\title{
Covariant integral quantization of the unit disk ${ }^{\circledR} P$
}

Cite as: J. Math. Phys. 61, 022101 (2020); https://doi.org/10.1063/1.5128066

Submitted: 16 September 2019 . Accepted: 19 January 2020 . Published Online: 21 February 2020

M. A. del Olmo (D), and J. P. Gazeau (D)

COLLECTIONS

EP This paper was selected as an Editor's Pick

\section{Journal of}

Mathematical Physics

Young Researcher Award

Recognizing the outstanding work of early career researchers 


\title{
Covariant integral quantization of the unit disk $\mathbb{\oplus}$
}

\author{
Cite as: J. Math. Phys. 61, 022101 (2020); doi: 10.1063/1.5128066 \\ Submitted: 16 September 2019 - Accepted: 19 January 2020 • \\ Published Online: 21 February 2020
}

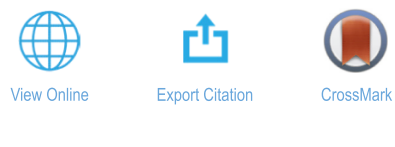

\begin{abstract}
M. A. del Olmo ${ }^{1, a)}$ and J. P. Gazeau ${ }^{2}$
AFFILIATIONS

${ }^{1}$ Departamento de Física Teórica, Atómica y Óptica and IMUVA - Mathematical Research Institute, Universidad de Valladolid, Paseo Belén 7, 47011 Valladolid, Spain

${ }^{2}$ Centro Brasileiro de Pesquisas Físicas, Rua Xavier Sigaud 150, 22290-180 Rio de Janeiro, RJ, Brazil and APC, UMR 7164, Université de Paris, CNRS, Astroparticule et Cosmologie, F-75013 Paris, France
\end{abstract}

a) marianoantonio.olmo@uva.es

b) Author to whom correspondence should be addressed: gazeau@apc.in2p3.fr

\begin{abstract}
We implement a $S U(1,1)$ covariant integral quantization of functions on the unit disk. The latter can be viewed as the phase space for the motion of a "massive" test particle on $(1+1)$-anti-de Sitter space-time, and the relevant unitary irreducible representations of SU(1,1) corresponding to the quantum version of such motions are found in the discrete series and its lower limit. Our quantization method depends on the choice of a weight function on the phase space in such a way that different weight functions yield different quantizations. For instance, the Perelomov coherent states quantization is derived from a particular choice. Semi-classical portraits or lower symbols of main physically relevant operators are determined, and the statistical meaning of the weight function is discussed.
\end{abstract}

Published under license by AIP Publishing. https://doi.org/10.1063/1.5128066

\section{INTRODUCTION}

The group SU $(1,1)$, which is the two-fold covering of $\mathrm{SO}_{0}(2,1)$, can be interpreted as the kinematical group for the $(1+1)$-anti-de-Sitter space-time. The unit disk $\mathscr{D}$ in the complex plane can be viewed as the phase space for the motion of an elementary system in this spacetime $^{1-4}$ [for the realistic $(1+3)$-anti-de-Sitter space-time with its kinematical group $\mathrm{SO}_{0}(2,3)$ and the relevant phase space $\mathrm{SO}_{0}(2,3) / \mathrm{SO}(2)$ $\times \mathrm{SO}(3)$, see Ref. 5 and references therein]. Therefore, it is appealing to carry out a comprehensive program of quantization of functions (or distributions) on the unit disk by using all resources of covariant integral quantization as it is defined, for instance, in Refs. 6-9 (and references therein). This program also includes the semi-classical return to the original phase space through the construction of the so-called lower ${ }^{10}$ or covariant ${ }^{11,12}$ symbols. The latter has a true probabilistic interpretation when the integral quantization is based on normalized positive operator valued measures (POVM) the latter acronym being used for normalized POVM as well.

Of course, due to the basic nature of the model, one can find in the literature different quantization methods which have been or could be applied to the unit disk. Besides the seminal Berezin's contributions, ${ }^{11,12}$ where the quantization of the unit disk is carried out through the notions of covariant and contravariant symbols built from SU $(1,1)$ coherent states in the Perelomov sense, ${ }^{13}$ there is the well-known Kirillov-Kostant-Souriau orbit method; see, for instance, Ref. 14 for a comprehensive review, and its parent, the geometric quantization. ${ }^{15,16}$ Deformation quantization ${ }^{17}$ is another popular method which has been applied to the unit disk in Ref. 18 , where a non-commutative $\mathbb{C}^{*}$ algebra from the algebra of the continuous function on the closed unit disk is obtained. For a general review of various quantization methods, see, for instance, Ref. 19.

To some extent, the approach followed in the present work pertains to the Berezin-Toeplitz quantization for Kähler manifolds, ${ }^{20-22}$ the unit disk being one of the simplest models. In a nutshell, the Berezin-Toeplitz quantization of a symplectic manifold $M$ with Kähler structure maps functions on $M$ to operators in the Hilbert space of square-integrable holomorphic sections of an appropriate complex line bundle. Denoting by $\Pi$ the orthogonal projection operator from the space of all square-integrable sections to the holomorphic subspace, for any bounded measurable function $f$, one constructs the Toeplitz operator $A_{f}$ with symbol $f$, acting on the space of holomorphic sections, as $A_{f} \phi=\Pi(f \phi)$. That is, $A_{f} \phi$ consists of multiplication by $f$ followed by projection back into the holomorphic subspace. This quantization map may be thought of as a generalization of anti-Wick-ordered quantization. The specificity and the novelty of our contribution are to 
be considered at various levels. First, we consider manifolds which are symmetric spaces derived from Cartan decomposition $G=P K$ of arbitrary semi-simple Lie groups, and we introduce a family of quantization projectors $\Pi$ derived from weight functions defined on the Cartan subalgebra $A \subset P$. Then, we apply the derived $G$-covariant integral quantization to the elementary case $G=\operatorname{SU}(1,1)$, for which the considered manifold is precisely Kählerian. While the Berezin approach ${ }^{11,12}$ to this example corresponds to a specific choice of the weight function, our article contains a set of original results due to the arbitrariness of the weight function. Moreover, the SU(1,1) example displays a rich set of properties which can be generalized to many Lie groups.

The organization of the paper is as follows. Sections II and III are a reminder of a known material about SU(1,1) as it can be found in a classical treatise in group representation theory like Ref. 23 or in the more recent Ref. 7. In Sec. II, we describe the geometry of the unit disk, viewed, respectively, as a Kälherian manifold in Subsection II A, as a left coset of SU $(1,1)$ in Subsection II B, with group action in the usual way, and, in Subsection II C, as the phase space for the motion of a test particle in $(1+1)$-anti-de Sitter space-time, with the identification of the three basic observables with SU $(1,1)$ generators. In Subsection II D, we complete these geometric and algebraic aspects with the description of the $(1+1)$-AdS space-time, which can be identified with a left coset of SU $(1,1)$. Section III is devoted to some representations of SU(1,1) relevant to our purposes. In Subsection III A, we give a concise description of the discrete series (in a wide sense) of representations of $\mathrm{SU}(1,1)$ as acting on Fock-Bargmann Hilbert spaces of holomorphic functions in the unit disk, and in Subsection III C, of their generators as first-order differential operators on these functions. In Sec. IV, we give a self-contained description of the framework of covariant integral quantization when it is associated with a unitary irreducible representation (UIR) of a Lie group in Subsection IV A, and with a squareintegrable UIR in Subsection IV B. (For more details and examples, see, for instance, Ref. 8 and references therein.) Then, in Subsection IV C, we implement this method with the restriction to the coset issued from the Cartan decomposition of the group and with the introduction of a weight function defined on a certain submanifold of the Cartan symmetric space. We also involve in the construction a parity operator as the representative in the UIR carrier Hilbert space of the Cartan involution. Together with the fact that different admissible weight functions lead to different quantizations, these features constitute an original aspect of the present work. In Sec. V, we apply the above formalism to the group $\mathrm{SU}(1,1)$, its discrete series, and the unit disk corresponding to the Cartan symmetric space, and we examine the outcomes when a particular family of weights is considered. In Subsection V C, we recover results obtained by other authors for specific weights of this family. We then proceed in Sec. VI with the quantization of functions on the AdS phase space (i.e., classical observables) and study the dependence of their respective quantized versions on the choice of the weight function, noticing that for the most basic ones, there is no or trivial dependence. Section VII is devoted to the lower symbols of operators issued from our covariant integral quantization. This amounts to give semi-classical portraits, in general, more regular, of the original function, together with a probabilistic interpretation when the weight function is suitably selected. In Sec. VIII, we conclude by commenting some aspects of our work amenable to further interesting developments. In the Appendix, some useful integral formulas involving Jacobi polynomials are given.

Most of our approaches should be justified on a mathematical level with regard to involved functions. Nevertheless, many of our results are given with implicit assumptions on their validity for the sake of simplicity. Throughout the text we use for convenience the shortened notation $f(z)$ in place of $f(z, \bar{z})$ for $z \in \mathbb{C}$, at the difference of Berezin in Refs. 11 and 12.

\section{GEOMETRY OF THE UNIT DISK AND ITS SYMMETRY}

\section{A. The unit disk as a Kählerian manifold}

The unit disk

$$
\mathscr{D} \stackrel{\text { def }}{=}\{z \in \mathbb{C},|z|<1\}
$$

is one of the four two-dimensional Kählerian manifolds, ${ }^{13,24}$ the other ones being, respectively, the complex plane $\mathbb{C}$, the sphere $\mathbb{S}^{2}$, or equivalently the projective complex line $\mathbb{C} \mathbb{P}^{1}$, and the torus $\mathbb{C} / \mathbb{Z}_{2} \sim \mathbb{S}^{1} \times \mathbb{S}^{1}$. It is equipped of the (Poincaré) metric,

$$
\mathrm{d} s^{2}=\frac{\mathrm{d} z \mathrm{~d} \bar{z}}{\left(1-|z|^{2}\right)^{2}}
$$

The corresponding surface element is given by the two-form,

$$
\Omega=\frac{i}{2} \frac{\mathrm{d} z \wedge \mathrm{d} \bar{z}}{\left(1-|z|^{2}\right)^{2}}=\frac{\mathrm{d}(\Re z) \mathrm{d}(\mathfrak{I} z)}{\left(1-|z|^{2}\right)^{2}} \equiv \frac{\mathrm{d}^{2} z}{\left(1-|z|^{2}\right)^{2}} \equiv \mu\left(\mathrm{d}^{2} z\right) .
$$

These quantities are both issued from a Kählerian potential $\mathscr{K}_{\mathscr{D}}$,

$$
\begin{aligned}
\mathscr{K}_{\mathscr{D}}(z, \bar{z}) & \stackrel{\text { def }}{=} \pi^{-1}\left(1-|z|^{2}\right)^{-2}, \\
\mathrm{~d} s^{2} & =\frac{1}{2} \frac{\partial^{2}}{\partial z \partial \bar{z}} \ln \mathscr{K}_{\mathscr{D}}(z, \bar{z}) d z d \bar{z}, \\
\mu\left(\mathrm{d}^{2} z\right) & =\frac{i}{4} \frac{\partial^{2}}{\partial z \partial \bar{z}} \ln \mathscr{K}_{\mathscr{D}}(z, \bar{z}) d z \wedge d \bar{z} .
\end{aligned}
$$




\section{B. The unit disk as a coset of $\operatorname{SU}(1,1)$}

Let us start by recalling the essential definitions and notations for the simple Lie group $\operatorname{SU}(1,1)$ and its Lie algebra,

$$
\mathrm{SU}(1,1)=\left\{g=\left(\begin{array}{ll}
\alpha & \beta \\
\bar{\beta} & \bar{\alpha}
\end{array}\right) \alpha, \beta \in \mathbb{C}, \operatorname{det} g=|\alpha|^{2}-|\beta|^{2}=1\right\} .
$$

The three basis elements of the Lie algebra $\mathfrak{s u}(1,1)$ are chosen as

$$
N_{0}=\frac{1}{2}\left(\begin{array}{rr}
\mathrm{i} & 0 \\
0 & -\mathrm{i}
\end{array}\right)=\frac{\mathrm{i} \sigma_{3}}{2}, N_{1}=\frac{1}{2}\left(\begin{array}{ll}
0 & 1 \\
1 & 0
\end{array}\right)=\frac{\sigma_{1}}{2}, \quad N_{2}=\frac{1}{2}\left(\begin{array}{rr}
0 & \mathrm{i} \\
-\mathrm{i} & 0
\end{array}\right)=-\frac{\sigma_{2}}{2},
$$

with the commutation relations

$$
\left[N_{0}, N_{1}\right]=N_{2}, \quad\left[N_{0}, N_{2}\right]=-N_{1}, \quad\left[N_{1}, N_{2}\right]=-N_{0}
$$

The Cartan factorization of $\mathrm{SU}(1,1)$ is associated with the (Cartan) involution

$$
i_{p h}: g \mapsto\left(g^{\dagger}\right)^{-1}
$$

The maximal compact subgroup $H=\mathrm{U}(1)$ is determined by $i_{p h}(g)=g$, whereas the condition $i_{p h}(g)=g^{-1}$ selects the subset $P$ of Hermitian matrices in $\mathrm{SU}(1,1)$. The factorization $\mathrm{SU}(1,1)=P H$ reads explicitly

$$
\mathrm{SU}(1,1) \ni g=\left(\begin{array}{cc}
\alpha & \beta \\
\bar{\beta} & \bar{\alpha}
\end{array}\right)=p(z) h(\theta)
$$

with

$$
p(z)=\left(\begin{array}{cc}
\delta & \delta z \\
\delta \bar{z} & \delta
\end{array}\right), \quad z=\beta \bar{\alpha}^{-1}, \quad \delta=|\alpha|=\left(1-|z|^{2}\right)^{-1 / 2}
$$

and

$$
h(\theta)=\left(\begin{array}{cc}
e^{i \theta / 2} & 0 \\
0 & e^{-i \theta / 2}
\end{array}\right), \quad \theta=2 \arg \alpha, \quad 0 \leq \theta<4 \pi .
$$

The bundle section $\mathscr{D} \in z \mapsto p(z) \in P$ gives the unit disk $\mathscr{D}$ a symmetric space realization identified as the coset space $\mathrm{SU}(1,1) / H$. Note that

$$
p^{2}=g g^{\dagger} \quad(p(z))^{-1}=p(-z) .
$$

The Haar measure (see, for instance, Ref. 23) on the unimodular group SU $(1,1)$ corresponding to the above Cartan factorization reads

$$
\mathrm{d}_{\text {haar }}(g)=\frac{1}{8 \pi^{2}} \frac{\mathrm{d}^{2} z}{\left(1-|z|^{2}\right)^{2}} \mathrm{~d} \theta \text {. }
$$

It is normalized for the angular parts.

The Cartan factorization allows us to make $\mathrm{SU}(1,1)$ act on $\mathscr{D}$ through a left action on the set of matrices $p(z)$,

$$
g: p(z) \mapsto p\left(z^{\prime}\right) \quad \text { with } \quad g p(z)=p\left(z^{\prime}\right) h^{\prime},
$$

where $z^{\prime} \equiv g . z$ is given by the map

$$
\begin{aligned}
\mathscr{D} \ni z \mapsto z^{\prime} & =(\alpha z+\beta)(\bar{\beta} z+\bar{\alpha})^{-1} \in \mathscr{D}, \\
& \Leftrightarrow z=\left(\bar{\alpha} z^{\prime}-\beta\right)\left(-\bar{\beta} z^{\prime}+\alpha\right)^{-1}=g^{-1} \cdot z^{\prime},
\end{aligned}
$$

and $h^{\prime}$ is the following element in $\mathrm{U}(1)$, 


$$
h^{\prime}=\left(\begin{array}{cc}
\frac{\beta \bar{z}+\alpha}{|\beta \bar{z}+\alpha|} & 0 \\
0 & \frac{\bar{\beta} z+\bar{\alpha}}{|\bar{\beta} z+\bar{\alpha}|}
\end{array}\right) \text {. }
$$

Note the formula used to derive the above relations,

$$
\delta(g \cdot z)=\delta(z)|\bar{\beta} z+\bar{\alpha}|^{-1},
$$

and also the action of $\mathrm{U}(1)$ on the disk,

$$
h(\theta) p(z) h(-\theta)=p(h(\theta) \cdot z)=p\left(e^{\mathrm{i} \theta z}\right) .
$$

Hence, the unit disk $\mathscr{D}$ is invariant under the transformations (8) of the homographic or Möbius type. Note that $\mathrm{SU}(1,1)$ leaves invariant the boundary $\mathbb{S}^{1} \simeq U(1)$ of $\mathscr{D}$ under the transformation (8).

The invariance of $\mathscr{D}$ under (8) also holds for the metric quantities (2),

$$
\begin{aligned}
\mathscr{K}_{\mathscr{O}}(z, \bar{z}) & =\pi^{-1}\left(1-|z|^{2}\right)^{-2}=\pi^{-1}\left(1-\left|z^{\prime}\right|^{2}\right)^{-2}, \\
\mathrm{~d} s^{2} & =\frac{\mathrm{d} z \mathrm{~d} \bar{z}}{\left(1-|z|^{2}\right)^{2}}=\frac{\mathrm{d} z^{\prime} \mathrm{d} \bar{z}^{\prime}}{\left(1-\left|z^{\prime}\right|^{2}\right)^{2}}, \\
\mu\left(\mathrm{d}^{2} z\right) & =\frac{\mathrm{d}(\Re z) \mathrm{d}(\Im z)}{\left(1-|z|^{2}\right)^{2}}=\frac{\mathrm{d}\left(\Re z^{\prime}\right) \mathrm{d}\left(\Im z^{\prime}\right)}{\left(1-\left|z^{\prime}\right|^{2}\right)^{2}} .
\end{aligned}
$$

\section{The unit disk as an AdS phase space}

Since the unit disk is Kählerian, it is symplectic and so can be given a phase space structure and related interpretation. First, the form (1) determines the Poisson bracket

$$
\{f, g\}=\frac{1}{2 \mathrm{i}}\left(1-|z|^{2}\right)^{2}\left(\frac{\partial f}{\partial z} \frac{\partial g}{\partial \bar{z}}-\frac{\partial f}{\partial \bar{z}} \frac{\partial g}{\partial z}\right) .
$$

Now, there are 3 basic observables generating the $\mathrm{SU}(1,1)$ symmetry on this classical level,

$$
\mathscr{D} \ni z \mapsto k_{0}(z)=\frac{1+|z|^{2}}{1-|z|^{2}}, k_{1}(z)=\frac{1}{\mathrm{i}} \frac{z-\bar{z}}{1-|z|^{2}}, k_{2}(z)=\frac{z+\bar{z}}{1-|z|^{2}} .
$$

They are not independent since

$$
k_{0}^{2}-k_{1}^{2}-k_{2}^{2}=1,
$$

i.e., the 3 -vector $\left(k_{0}, k_{1}, k_{2}\right)$ points to the upper sheet $\mathscr{H}_{+}$of the two-sheeted hyperboloid in $\mathbb{R}^{3}$ which is described by (11), and whose stereographic projection through (10) is the open unit disk. This projection reads

$$
\mathscr{H}_{+} \ni\left(k_{0}, k_{1}, k_{2}\right) \mapsto z=\frac{k_{2}+\mathrm{i} k_{1}}{1+k_{0}} \equiv \sqrt{\frac{k_{0}-1}{k_{0}+1}} e^{\mathrm{i} \arg z} .
$$

They obey the Poisson commutation rules

$$
\left\{k_{0}, k_{1}\right\}=k_{2}, \quad\left\{k_{0}, k_{2}\right\}=-k_{1}, \quad\left\{k_{1}, k_{2}\right\}=-k_{0},
$$

which are consistent with (4). As is expected, the two combinations

$$
k_{+}=k_{2}-\mathrm{i} k_{1}=\frac{2 \bar{z}}{1-|z|^{2}}, \quad k_{-}=k_{2}+\mathrm{i} k_{1}=\frac{2 z}{1-|z|^{2}},
$$

are to play an important role as well.

Under the action of $g=\left(\begin{array}{cc}\alpha & \beta \\ \bar{\beta} & \bar{\alpha}\end{array}\right) \in \mathrm{SU}(1,1)$, functions $k_{0}$ and $k_{ \pm}$transform as

$$
\begin{aligned}
& k_{0}^{\prime}(z)=k_{0}\left(g^{-1} \cdot z\right)=\left(|\alpha|^{2}+|\beta|^{2}\right) k_{0}(z)-2 \Re\left(\alpha \beta k_{+}(z)\right), \\
& k_{+}^{\prime}(z)=k_{+}\left(g^{-1} \cdot z\right)=-2 \alpha \bar{\beta} k_{0}(z)+\alpha^{2} k_{+}(z)+\bar{\beta}^{2} k_{-}(z), \\
& k_{-}^{\prime}(z)=k_{-}\left(g^{-1} \cdot z\right)=-2 \bar{\alpha} \beta k_{0}(z)+\bar{\alpha}^{2} k_{-}(z)+\beta^{2} k_{+}(z)=\overline{k_{+}\left(g^{-1} \cdot z\right)} .
\end{aligned}
$$


Equivalently, in matrix notation,

$$
\left(\begin{array}{cc}
k_{-}^{\prime} & k_{0}^{\prime} \\
k_{0}^{\prime} & k_{+}^{\prime}
\end{array}\right)=g^{-1}\left(\begin{array}{ll}
k_{-} & k_{0} \\
k_{0} & k_{+}
\end{array}\right)\left(g^{-1}\right)^{t} .
$$

This transform can be viewed as the (co-adjoint action) of SU $(1,1)$ on the co-adjoint orbit identified with (11). Introducing the left action of $\mathrm{SU}(1,1)$ on functions $f(z)$ as

$$
(\mathfrak{U}(g) f)(z):=f\left(g^{-1} \cdot z\right)
$$

we write the above transformations in terms of matrix elements as

$$
\left(\mathfrak{U}(g) k_{a}\right)(z)=\sum_{b}[\mathfrak{U}(g)]_{b a} k_{b}(z), \quad a=0,1,2 \text { or } a=0, \pm .
$$

The following particularization of these formulas to $g=p\left(-z^{\prime}\right)$ will be useful for Sec. VII:

$$
\begin{aligned}
& k_{0}\left(p\left(z^{\prime}\right) \cdot z\right)=k_{0}\left(z^{\prime}\right) k_{0}(z)+\Re\left(k_{-}\left(z^{\prime}\right) k_{+}(z)\right), \\
& k_{+}\left(p\left(z^{\prime}\right) \cdot z\right)=k_{+}\left(z^{\prime}\right) k_{0}(z)+\left(1-\left|z^{\prime}\right|^{2}\right)\left(k_{+}(z)+{\overline{z^{\prime}}}^{2} k_{-}(z)\right), \\
& k_{-}\left(p\left(z^{\prime}\right) \cdot z\right)=\overline{k_{+}\left(p\left(z^{\prime}\right) \cdot z\right) .}
\end{aligned}
$$

\section{AdS space-time as a left coset of $S U(1,1)$}

$$
g=h(\theta) s(u) l(v), \quad \theta \in[0,2 \pi), u, v \in \mathbb{R} .
$$

Whereas the first factor

$$
h(\theta)=\epsilon\left(\begin{array}{cc}
e^{\mathrm{i} \theta / 2} & 0 \\
0 & e^{-\mathrm{i} \theta / 2}
\end{array}\right), \quad \epsilon= \pm I_{2},
$$

belongs to $\mathrm{U}(1)$, the maximal compact subgroup, with $\epsilon$ belonging to the center of $\mathrm{SU}(1,1)$ isomorphic to $\mathbb{Z}_{2}$, and the others are of noncompact hyperbolic type,

$$
s(u)=\left(\begin{array}{rr}
\cosh \frac{u}{2} & \sinh \frac{u}{2} \\
\sinh \frac{u}{2} & \cosh \frac{u}{2}
\end{array}\right), \quad l(v)=\left(\begin{array}{rr}
\cosh \frac{v}{2} & \text { i } \sinh \frac{v}{2} \\
-i \sinh \frac{v}{2} & \cosh \frac{v}{2}
\end{array}\right), \quad u, v \in \mathbb{R}
$$

and belong to subgroups isomorphic to $\mathbb{R}$. Their respective generators $N_{a}, a=0,1,2$, are precisely those introduced in (3),

$$
h(\theta)=\epsilon e^{\theta N_{0}}, \quad s(u)=e^{u N_{1}}, \quad l(v)=e^{v N_{2}}
$$

The factorization (17) is associated with the group involution

$$
i_{j}: g \mapsto g^{t},
$$

where the superscript $t$ denotes transposition. Indeed,

$$
h^{t}=h, \quad s^{t}=s, \quad l^{t}=l^{-1} .
$$

Now, by considering

$$
j(\theta, u)=h(\theta) s(u)=\left(\begin{array}{cc}
e^{\mathrm{i} \theta / 2} \cosh \frac{u}{2} & e^{\mathrm{i} \theta / 2} \sinh \frac{u}{2} \\
e^{-\mathrm{i} \theta / 2} \sinh \frac{u}{2} & e^{-\mathrm{i} \theta / 2} \cosh \frac{u}{2}
\end{array}\right),
$$

we have 


$$
j j^{t}=g g^{t}=\left(\begin{array}{cc}
\alpha^{2}+\beta^{2} & 2 \Re(\alpha \bar{\beta}) \\
2 \Re(\alpha \bar{\beta}) & \bar{\alpha}^{2}+\bar{\beta}^{2}
\end{array}\right)=\left(\begin{array}{cc}
e^{\mathrm{i} \theta} \cosh u & \sinh u \\
\sinh u & e^{-\mathrm{i} \theta} \cosh u
\end{array}\right) .
$$

The parameters $(\theta, u)$ form a system of global coordinates for the $(1+1)$-anti-de Sitter space-time visualized as the one-sheeted hyperboloid $\eta_{a b} y^{a} y^{b}=\kappa^{-2}$ in $\mathbb{R}^{3}$ with metric $\left(\eta^{a b}\right)=\operatorname{diag}(+,+,-), a, b=2,0,1$, and with curvature $\kappa$,

$$
\begin{aligned}
& y^{2}=\kappa^{-1} \cosh u \cos \theta \\
& y^{0}=\kappa^{-1} \cosh u \sin \theta \\
& y^{1}=\kappa^{-1} \sinh u
\end{aligned}
$$

or expressed in terms of the element of $\operatorname{SU}(1,1)$,

$$
j j^{t}=\left(\begin{array}{cc}
\kappa y_{+} & \kappa y^{1} \\
\kappa y^{1} & \kappa y_{-}
\end{array}\right) \equiv \Gamma(y),
$$

such that

$$
y_{ \pm}=y^{2} \pm \mathrm{i} y^{0}, \quad \kappa^{-2} \operatorname{det} \Gamma(y)=\eta_{a b} y^{a} y^{b}=\kappa^{-2} .
$$

The factorization $g=j l$, which means on the group level $\mathrm{SU}(1,1)=$ AdS $\times$ Lorentz, allows us to view the AdS space-time as the left coset $\mathrm{SU}(1,1) / \mathrm{L}$, with $\mathrm{L}=\{l(v), v \in \mathbb{R}\} \sim \mathrm{SO}_{0}(1,1)$ being the orthochronous Lorentz subgroup. $\mathrm{SU}(1,1)$ acts on the set of matrices $\Gamma(y)$, and this action is induced from its left action on the set of matrices $j$,

$$
g: j \mapsto j^{\prime}, g j=j^{\prime} l^{\prime} \Leftrightarrow \Gamma\left(y^{\prime}\right)=j^{\prime} j^{t}=g j j^{t} g^{t}=g \Gamma(y) g^{t} .
$$

In this interpretation, $\mathrm{SU}(1,1)$ acts as the double covering of the actual AdS group $\mathrm{SO}_{0}(2,1)=\mathrm{SU}(1,1) / \mathbb{Z}_{2}$, and we see that $N_{0}$ generates the "translations in time" corresponding to $U(1), N_{1}$ generates the "translations in space" corresponding to the subgroup $\mathrm{SO}_{0}(1,1)$, and $N_{2}$ generates the Lorentz transformations corresponding to the other $\mathrm{SO}_{0}(1,1)=\mathrm{L}$.

It is instructive to describe how the three basic observables $k_{a}, a=0,1,2$ (or $k_{0}, k_{ \pm}$), transform under the action of three subgroups with respective generators $N_{a}, a=0,1,2$,

$$
\begin{aligned}
& h(\theta):\left\{\begin{array}{l}
k_{0} \mapsto k_{0}, \\
k_{ \pm} \mapsto e^{ \pm i \theta} k_{ \pm},
\end{array}\right. \\
& s(u):\left\{\begin{array}{l}
k_{0} \mapsto \cosh u k_{0}-\sinh u k_{2}, \\
k_{1} \mapsto k_{1}, \\
k_{2} \mapsto-\sinh u k_{0}+\cosh u k_{2},
\end{array}\right. \\
& l(v):\left\{\begin{array}{l}
k_{0} \mapsto \cosh v k_{0}-\sinh v k_{1}, \\
k_{1} \mapsto-\sinh v k_{0}+\cosh u k_{1}, \\
k_{2} \mapsto k_{2} .
\end{array}\right.
\end{aligned}
$$

\section{SU(1, 1) REPRESENTATION(S)}

\section{A. $S U(1,1)$ unitary irreducible representation(s) (discrete series)}

For a given $\eta>1 / 2$, consider the Fock-Bargmann Hilbert space $\mathscr{F} \mathscr{B} \eta$ of all analytic functions $f(z)$ on $\mathscr{D}$ that are square integrable with respect to the scalar product,

$$
\left\langle f_{1} \mid f_{2}\right\rangle=\frac{2 \eta-1}{2 \pi} \int_{\mathscr{D}} \overline{f_{1}(z)} f_{2}(z)\left(1-|z|^{2}\right)^{2 \eta-2} \mathrm{~d}^{2} z
$$

An orthonormal basis is made of powers of $z$ suitably normalized,

$$
e_{n}(z) \equiv \sqrt{\frac{(2 \eta)_{n}}{n !}} z^{n} \quad \text { with } \quad n \in \mathbb{N},
$$

where $(2 \eta)_{n}:=\Gamma(2 \eta+n) / \Gamma(2 \eta)$ is the Pochhammer symbol. For $\eta=1,3 / 2,2,5 / 2, \ldots$, one defines the UIR $g=\left(\begin{array}{cc}\alpha & \beta \\ \bar{\beta} & \bar{\alpha}\end{array}\right) \mapsto U^{\eta}(g)$ of $S U(1,1)$ on $\mathscr{F} \mathscr{B}_{\eta}$ by 


$$
\mathscr{F} \mathscr{B}_{\eta} \ni f(z) \mapsto\left(U^{\eta}(g) f\right)(z)=(-\bar{\beta} z+\alpha)^{-2 \eta} f\left(\frac{\bar{\alpha} z-\beta}{-\bar{\beta} z+\alpha}\right) .
$$

In particular, for $g=p\left(z^{\prime}\right)$, we have

$$
\left(U^{\eta}\left(p\left(z^{\prime}\right)\right) f\right)(z)=\left(1-\left|z^{\prime}\right|^{2}\right)^{\eta}\left(1-z \bar{z}^{\prime}\right)^{-2 \eta} f\left(\frac{z-z^{\prime}}{1-z \bar{z}^{\prime}}\right) .
$$

This countable set of representations constitutes the "almost complete" holomorphic discrete series of representations of SU(1,1).23 "Almost complete" because the lowest one, $\eta=1 / 2$, requires a special treatment due to the non-existence of the inner product (18) in this case. Had we considered the continuous set $\eta \in[1 / 2,+\infty)$, we would have been led to involve the universal covering of $S U(1,1)$.

The matrix elements of the operator $U^{\eta}(g)$ with respect to the orthonormal basis (19) are given in terms of hypergeometric polynomials by ${ }^{25}$

$$
\begin{aligned}
U_{n n^{\prime}}^{\eta}(g)=\left\langle e_{n}\left|U^{\eta}(g)\right| e_{n^{\prime}}\right\rangle=\left(\frac{n_{>} ! \Gamma\left(2 \eta+n_{>}\right)}{n_{<} ! \Gamma\left(2 \eta+n_{<}\right)}\right)^{1 / 2} \alpha^{-2 \eta-n_{>}} \bar{\alpha}^{n_{<}} \\
\quad \quad \times \frac{(\gamma(\beta, \bar{\beta}))^{n_{>}-n_{<}}}{\left(n_{>}-n_{<}\right) !}{ }_{2} F_{1}\left(-n_{<}, n_{>}+2 \eta ; n_{>}-n_{<}+1 ; \frac{|\beta|^{2}}{|\alpha|^{2}}\right),
\end{aligned}
$$

where

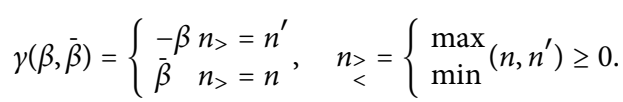

Taking into account the well-known relation between the hypergeometric functions and the Jacobi ${ }^{26}$ polynomials,

$$
P_{n}^{(\mu, v)}(x)=\left(\begin{array}{c}
n+\mu \\
n
\end{array}\right){ }_{2} F_{1}\left(-n, n+\mu+v+1 ; \mu+1 ; \frac{1-x}{2}\right)
$$

and the parametrization (5), this expression is alternatively given in terms of Jacobi polynomials as

$$
\begin{aligned}
U_{n n^{\prime}}^{\eta}(g)=\left(\frac{n_{<} ! \Gamma\left(2 \eta+n_{>}\right)}{n_{>} ! \Gamma\left(2 \eta+n_{<}\right)}\right)^{1 / 2} \alpha^{-2 \eta-n_{>}} \bar{\alpha}^{n_{<}} \\
\quad \times(\gamma(\beta, \bar{\beta}))^{n_{>}-n_{<}} P_{n_{<}}^{\left(n_{>}-n_{<}, 2 \eta-1\right)}\left(1-2|z|^{2}\right), \quad z=\beta \bar{\alpha}^{-1} .
\end{aligned}
$$

Note the diagonal elements,

$$
U_{n n}^{\eta}(g)=\alpha^{-2 \eta-n} \bar{\alpha}_{2}^{n} F_{1}\left(-n, n+2 \eta ; 1 ; \frac{|\beta|^{2}}{|\alpha|^{2}}\right)=\alpha^{-2 \eta}\left(\frac{\bar{\alpha}}{\alpha}\right)^{n} P_{n}^{(0,2 \eta-1)}\left(1-2|z|^{2}\right) .
$$

For the elements $g=h(\theta)$ in $\mathrm{U}(1)$, we have

$$
U_{n n^{\prime}}^{\eta}(h(\theta))=\delta_{n n^{\prime}} e^{-\mathrm{i}(\eta+n) \theta}
$$

whereas for the elements $g=p(z)$ in $P$,

$$
\begin{aligned}
& U_{n n^{\prime}}^{\eta}(p(z))=\left(\frac{n_{>} ! \Gamma(2 \eta}{n_{<} ! \Gamma\left(2 \eta+n_{>}\right)}\right) n^{1 / 2}\left(1-|z|^{2}\right)^{\eta} \frac{|z|^{n_{>}-n_{<}}}{\left(n_{>}-n_{<}\right) !} e^{\mathrm{i}\left(n^{\prime}-n\right) \phi} \times \\
& \times\left(\operatorname{sgn}\left(n-n^{\prime}\right)\right)^{n-n^{\prime}}{ }_{2} F_{1}\left(-n_{<}, n_{>}+2 \eta ; n_{>}-n_{<}+1 ;|z|^{2}\right) \\
&=\left(\frac{n_{<} ! \Gamma\left(2 \eta+n_{>}\right)}{n_{>} ! \Gamma\left(2 \eta+n_{<}\right)}\right)^{1 / 2}\left(1-|z|^{2}\right)^{\eta}|z|^{n_{>}-n_{<}} e^{\mathrm{i}\left(n^{\prime}-n\right) \phi} \times \\
& \times\left(\operatorname{sgn}\left(n-n^{\prime}\right)\right)^{n-n^{\prime}} P_{n_{<}}^{\left(n_{>}-n_{<}, 2 \eta-1\right)}\left(1-2|z|^{2}\right),
\end{aligned}
$$

with $z=|z| e^{\mathrm{i} \phi}$. If $n=n^{\prime}$,

$$
U_{n n}^{\eta}(p(z))=\left(1-|z|^{2}\right)^{\eta}{ }_{2} F_{1}\left(-n, n+2 \eta ; 1 ;|z|^{2}\right)=\left(1-|z|^{2}\right)^{\eta} P_{n}^{(0,2 \eta-1)}\left(1-2|z|^{2}\right) .
$$




\section{B. Orthogonality relations and trace formulas}

Since the functions (21) are matrix elements of operators $U^{\eta}$ in the discrete series for $\eta>1 / 2$, one of their fundamental properties is displayed by their orthogonality relations,

$$
\int_{\mathrm{SU}(1,1)} \mathrm{d}_{\text {haar }}(g) U_{m m^{\prime}}^{\eta}(g) \overline{U_{n n^{\prime}}^{\eta}(g)}=d_{\eta} \delta_{m n} \delta_{m^{\prime} n^{\prime}},
$$

where $d_{\eta}=2 \pi /(2 \eta-1)$ is the (formal) dimension of the representation $U^{\eta}$.

From the extension of the generating function of Jacobi polynomials, ${ }^{26}$

$$
\sum_{n=0}^{\infty} P_{n}^{(\mu, v)}(x) t^{n}=\frac{2^{\mu+v}}{R}(1-t+R)^{-\mu}(1+t+R)^{-v}, \quad|t|<1, R=\left(1-2 t x+t^{2}\right)^{1 / 2},
$$

to its "forbidden" limit $|t|=1$, we infer the trace of the operator $U^{\eta}(g)$ for a general $g=\left(\begin{array}{cc}\alpha & \beta \\ \bar{\beta} & \bar{\alpha}\end{array}\right) \in \mathrm{SU}(1,1)$,

$$
\operatorname{tr}\left(U^{\eta}(g)\right)=\frac{1}{2}\left((\Re \alpha)^{2}-1\right)^{-1 / 2}\left[(\Re \alpha)^{2}+\left((\Re \alpha)^{2}-1\right)^{1 / 2}\right]^{1-2 \eta},
$$

and its restriction to $p(z)$,

$$
\operatorname{tr}\left(U^{\eta}(p(z))\right)=\frac{1}{2|z|}\left(1-|z|^{2}\right)^{\eta}(1+|z|)^{1-2 \eta}
$$

These expressions are singular for $\alpha=1$ and $z=0$, respectively, since these values correspond to the identity operator.

Another trace formula will play an important role in the sequel. It involves the parity operator defined by

$$
\begin{gathered}
\mathrm{P}:=\sum_{n=0}^{\infty}(-1)^{n}\left|e_{n}\right\rangle\left\langle e_{n}\right|, \\
\operatorname{tr}\left(\mathrm{P} U^{\eta}(g)\right)=\frac{1}{2}\left((1-\Im \alpha)^{2}\right)^{-1 / 2}\left[(\Im \alpha)^{2}+\left((1-\Im \alpha)^{2}\right)^{1 / 2}\right]^{1-2 \eta},
\end{gathered}
$$

and its restriction to $p(z)$,

$$
\operatorname{tr}\left(\mathrm{P} U^{\eta}(p(z))\right)=\frac{1}{2}
$$

For $z=0$, this gives a trace formula for the parity operator

$$
\operatorname{tr} \mathrm{P}=\sum_{k=0}^{\infty}(-1)^{k}=\frac{1}{2},
$$

which can be legitimated by adopting the Abel summation of divergent series.

Finally, the following formula (related to the existence of the so-called inversion in Cartan symmetric domains) will be used in this paper,

$$
\mathrm{P} U^{\eta}(p(z)) \mathrm{P}=U^{\eta}(p(-z)) \text {. }
$$

\section{Corresponding representation of the Lie algebra $\mathfrak{s u}(1,1)$}

The respective self-adjoint representatives of $N_{0}, N_{1}$, and $N_{2}$, defined in (3), under the UIR (20), defined generically asi $\partial U^{\eta}(g(t)) /\left.\partial t\right|_{t=0}$, are the following differential operators on the Fock-Bargmann space $\mathscr{F} \mathscr{B} \eta$ :

$$
\begin{aligned}
& N_{0} \mapsto K_{0}=z \frac{\mathrm{d}}{\mathrm{d} z}+\eta, \\
& N_{1} \mapsto K_{1}=-\frac{\mathrm{i}}{2}\left(1-z^{2}\right) \frac{\mathrm{d}}{\mathrm{d} z}+\mathrm{i} \eta z, \\
& N_{2} \mapsto K_{2}=\frac{1}{2}\left(1+z^{2}\right) \frac{\mathrm{d}}{\mathrm{d} z}+\eta z .
\end{aligned}
$$

They obey the commutation rules, 


$$
\left[K_{0}, K_{1}\right]=\mathrm{i} K_{2}, \quad\left[K_{0}, K_{2}\right]=-\mathrm{i} K_{1}, \quad\left[K_{1}, K_{2}\right]=-\mathrm{i} K_{0} .
$$

The elements of the orthonormal basis (19) are eigenvectors of the compact generator $K_{0}$ with equally spaced eigenvalues,

$$
K_{0}\left|e_{n}\right\rangle=(\eta+n)\left|e_{n}\right\rangle .
$$

The particular element $\left|e_{0}\right\rangle$ of the basis is a lowest weight state or "vacuum" for the representations $U^{\eta}$. Indeed, introducing the two operators with their commutation relation,

$$
K_{ \pm}=\mp \mathrm{i}\left(K_{1} \pm \mathrm{i} K_{2}\right)=K_{2} \mp \mathrm{i} K_{1}, \quad\left[K_{+}, K_{-}\right]=-2 K_{0} .
$$

As differential operators, they read as $K_{+}=z^{2} \mathrm{~d} / \mathrm{d} z+2 \eta z, K_{-}=\mathrm{d} / \mathrm{d} z$. Adjoint of each other, they are raising and lowering operators, respectively,

$$
\begin{aligned}
& K_{+}\left|e_{n}\right\rangle=\sqrt{(n+1)(2 \eta+n)}\left|e_{n+1}\right\rangle, \\
& K_{-}\left|e_{n}\right\rangle=\sqrt{n(2 \eta+n-1)}\left|e_{n-1}\right\rangle,
\end{aligned}
$$

and we check $K_{-}\left|e_{0}\right\rangle=0$. States $\left|e_{n}\right\rangle$ are themselves obtained by successive ladder actions on the lowest state as follows:

$$
\left|e_{n}\right\rangle=\sqrt{\frac{\Gamma(2 \eta)}{\Gamma(2 \eta+n) n !}}\left(K_{+}\right)^{n}\left|e_{0}\right\rangle
$$

The Casimir operator is defined as

$$
\mathscr{C} \stackrel{\text { def }}{=} K_{1}^{2}+K_{2}^{2}-K_{0}^{2}=\frac{K_{+} K_{-}+K_{-} K_{+}}{2}-K_{0}^{2} .
$$

This operator is fixed at the value $\mathscr{C}=-\eta(\eta-1) I_{d}$ on the space $\mathscr{F} \mathscr{B}_{\eta}$ that carries the UIR $U^{\eta}$.

Finally, we note that, in agreement with the covariance properties (12) of their respective classical counterparts, we have

$$
\begin{aligned}
U^{\eta}(g) K_{0} U^{\eta}\left(g^{-1}\right) & =\left(|\alpha|^{2}+|\beta|^{2}\right) K_{0}-\alpha \beta K_{+}-\bar{\alpha} \bar{\beta} K_{-}, \\
U^{\eta}(g) K_{+} U^{\eta}\left(g^{-1}\right) & =-2 \alpha \bar{\beta} K_{0}+\alpha^{2} K_{+}+\bar{\beta}^{2} K_{-}, \\
U^{\eta}(g) K_{-} U^{\eta}\left(g^{-1}\right) & =-2 \bar{\alpha} \beta K_{0}+\bar{\alpha}^{2} K_{-}+\beta^{2} K_{+} .
\end{aligned}
$$

Equivalently, with the notations of (15),

$$
U^{\eta}(g) K_{a} U^{\eta}\left(g^{-1}\right)=\sum_{b}[\mathfrak{U}(g)]_{b a} K_{b}(z), \quad a=0,1,2 \text { or } a=0, \pm .
$$

Like for the Weyl-Heisenberg group, a unitary "displacement" operator is built from the generators $K_{ \pm}$. It corresponds to map $\mathscr{D} \ni z \mapsto \xi \in \mathbb{C}$ determined by

$$
U^{\eta}(p(\bar{z}))=e^{\xi K_{+}-\bar{\xi} K_{-}} \equiv D_{\eta}(\xi), \quad \xi=\tanh ^{-1}|z| e^{\mathrm{i} \arg z},
$$

which gives $k_{0}(z)=\cosh 2|\xi|$ for the observable introduced in (10).

\section{COVARIANT INTEGRAL QUANTIZATIONS: GENERAL}

\section{A. Covariant integral quantization with UIR of a group}

Here, we give a self-contained presentation of the method (for more details and applications, see Refs. 6-9). Let $G$ be a locally compact Lie group with left Haar measure $\mathrm{d}_{\text {haar }}(g)$, and let $g \mapsto U(g)$ be a UIR of $G$ in a Hilbert space $\mathscr{H}$. Let M be a bounded operator on $\mathscr{H}$ and let us introduce the family

$$
\left\{M(g):=U(g) M U^{\dagger}(g), g \in G\right\}
$$

of the "displaced" version of $M$ under the action of $U(g)$ 's. Suppose that the operator,

$$
R:=\int_{G} M(g) d_{\text {haar }}(g),
$$

is defined in a weak sense. From the left invariance of $\mathrm{d}_{\text {haar }}(g)$, the operator $R$ commutes with all operators $U(g), g \in G$, and so, from Schur's Lemma, we have the "resolution" of the unity up to a constant,

$$
R=c_{\mathrm{M}} I
$$


with

$$
c_{M}=\int_{G} \operatorname{tr}\left(\rho_{0} M(g)\right) d_{\text {haar }}(g) .
$$

Here, the unit trace positive operator $\rho_{0}$ is chosen, if manageable, in order to make the integral convergent. Of course, it is possible that no such finite constant exists for the given $M$, and at worst, it could not exist for any $M$ (which is not the case for square integrable representations). Now, if $c_{M}$ is finite and positive, the true resolution of the identity follows:

$$
\int_{G} \mathrm{M}(g) \mathrm{d} v(g)=I, \quad \mathrm{~d} v(g):=\mathrm{d}_{\text {haar }}(g) / \mathcal{c}_{\mathrm{M}} .
$$

\section{B. Covariant integral quantization: With square integrable UIR}

Let us consider a UIR $U$ for which $M$ is an "admissible" operator, which means that

$$
c_{\mathrm{M}}=\int_{G} \mathrm{~d}_{\text {haar }}(g) \operatorname{tr}\left(\rho_{0} \mathrm{M}(g)\right)
$$

is finite for a certain $\rho_{0}$, or more specifically, for square-integrable UIR $U$ for which $M=\rho$ is an admissible density operator,

$$
c(\rho)=\int_{G} \mathrm{~d}_{\text {haar }}(g)\|\rho U(g)\|_{\mathscr{H} \mathscr{S}}^{2}<\infty,
$$

where $\|A\|_{\text {YeS }}=\operatorname{tr}\left(A A^{\dagger}\right)$ is the Hilbert-Schmidt norm. Then, the resolution of the identity is guaranteed with the family,

$$
M(g)=U(g) M U^{\dagger}(g)
$$

This allows the covariant integral quantization of complex-valued functions on the group

$$
f \mapsto A_{f}=\int_{G} M(g) f(g) \mathrm{d} v(g), \quad \mathrm{d} v(g)=\frac{\mathrm{d}_{\text {haar }}(g)}{c_{M}} \text { or } \frac{\mathrm{d}_{\text {haar }}(g)}{c(\rho)} .
$$

Covariance means that

$$
U(g) A_{f} U^{\dagger}(g)=A_{U_{\mathrm{reg}}(g) f},
$$

where

$$
\left(U_{\text {reg }}(g) f\right)\left(g^{\prime}\right):=f\left(g^{-1} g^{\prime}\right)
$$

is the regular representation if $f \in L^{2}\left(G, \mathrm{~d}_{\text {haar }}(g)\right.$ ). Moreover, we get a generalization of the Berezin or heat kernel transform on $G$ (see, for instance, Ref. 27),

$$
f(g) \mapsto \check{f}(g):=\int_{G} \operatorname{tr}\left(\rho(g) \rho\left(g^{\prime}\right)\right) f\left(g^{\prime}\right) \mathrm{d} v\left(g^{\prime}\right),
$$

where the function $\check{f}$ is the lower or covariant symbol of the operator $A_{f}$.

\section{Covariant integral quantization through Cartan and Iwasawa decomposition: The general case}

\section{Cartan and Iwasawa decompositions: A reminder}

Let $G$ be a connected semi-simple Lie group and $K$ its maximal compact subgroup. Then, the homogeneous coset space,

$$
G / K=\{\bar{g}, g \in G\}, \quad \bar{g}=\{g k, k \in K\},
$$

is symmetric (i.e., it is a smooth manifold whose group of symmetries contains an inversion symmetry about every point; for more details, see Ref. 28), diffeomorphic to an Euclidean space, and the Cartan decomposition,

$$
\begin{gathered}
G=P K \Leftrightarrow \forall g \in G \exists p \in P, k \in K, \\
\text { such that } g=p k=k p^{\prime}, p^{\prime}=k^{-1} p k,
\end{gathered}
$$

holds. The subset $P$ is in one-to-one correspondence with the left coset $G / K$. The decomposition (34) exponentiates the Lie algebra decomposition $\mathfrak{g}=\mathfrak{p}+\mathfrak{k}$, such that 


$$
[\mathfrak{k}, \mathfrak{k}] \subset \mathfrak{k}, \quad[\mathfrak{k}, \mathfrak{p}] \subset \mathfrak{p}, \quad[\mathfrak{p}, \mathfrak{p}] \subset \mathfrak{k} .
$$

The decomposition (34) derives from the Cartan involution $\vartheta$ defined on the group level by

$$
\vartheta(p)=p^{-1} \forall p \in P, \quad \vartheta(k)=k \forall k \in K,
$$

and on the Lie algebra level $(\mathfrak{g}=\mathfrak{p}+\mathfrak{k})$ by

$$
\vartheta(\mathfrak{p})=-\mathfrak{p}, \quad \vartheta(\mathfrak{k})=\mathfrak{k} .
$$

A derived decomposition involves the abelian subgroup $A=\exp \mathfrak{a}$, where $\mathfrak{a}$ is a (Cartan) maximal abelian subalgebra in $\mathfrak{p}$,

$$
G=K A K \text {. }
$$

When restricted to $P$, it can be refined as

$$
P=\bigcup_{k \in K} \operatorname{Ad} k \cdot A
$$

Geometrically, the image of the subgroup $A$ in $G / K$ is a totally geodesic submanifold.

We also need to consider the Iwasawa decomposition of the group $G$ which reads as

$$
G=N A K,
$$

where $N$ is the subgroup generated by the nilpotent Lie algebra $\mathfrak{n}$ given as the sum of the positive roots of $\mathfrak{a}$.

Since $G$ is unimodular, the Haar measure is left and right invariant. From the theory of homogeneous spaces, it can be factorized as

$$
\mathrm{d}_{\text {haar }}(g)=\mathrm{d} \mu_{G / K}(\bar{g}) \mathrm{d}_{\text {haar }}(k)
$$

in such a way that

$$
\int_{G} f(g) \mathrm{d}_{\text {haar }}(g)=\int_{G / K} \mathrm{~d} \mu_{G / K}(\bar{g}) \int_{K} \mathrm{~d}_{\text {haar }}(k) f(g k) .
$$

This has to be compared with the integration on the group resulting from its Iwasawa decomposition (37),

$$
\int_{G} f(g) \mathrm{d}_{\text {haar }}(g)=\int_{N} \int_{A} \int_{K} f(n a k) \mathrm{d}_{\text {haar }}(n) \mathrm{d}_{\text {haar }}(a) \mathrm{d}_{\text {haar }}(k) .
$$

There results

$$
\mathrm{d} \mu_{G / K}(\bar{g})=\mathrm{d}_{\text {haar }}(n) \mathrm{d}_{\text {haar }}(a) \quad \text { for } g=n a k, n \in N, a \in A, k \in K .
$$

Due to the one-to-one correspondence between $P$ and $G / K$, we will write as well

$$
\mathrm{d} \mu_{G / K}(\bar{g}) \equiv \mathrm{d} \mu_{P}(p)
$$

The action $g: \bar{g}_{0} \mapsto g \cdot \bar{g}_{0}=\overline{g g_{0}}$ of $G$ on $G / K$ is equivalently carried out through the left action on $P, g: p \mapsto p^{\prime}$ defined by $g p=p^{\prime} k^{\prime}$. Hence, the subgroup $K$ is the stabilizer of a point in $P$.

Based on the Iwasawa decomposition, let us denote by $\boldsymbol{w}=(v, \alpha)$ a set of coordinates for the manifold $G / K$ or, equivalently, for $P$, on which the left action of $G$ on $P$ is described as

$$
g: p \mapsto p^{\prime} \Leftrightarrow p^{\prime}(\boldsymbol{w}):=p(g \cdot w)
$$

This formula generalizes (7).

\section{Integral quantization of Cartan symmetric space}

Let $g \mapsto U(g)$ be a UIR of $G$. Suppose that we can generalize (28) by defining on the representation Hilbert space $\mathscr{H}$ of $U$ a bounded self-adjoint operator $\mathrm{P}$ obeying $\mathrm{P}^{2}=I$ and

$$
\mathrm{P} U(p) \mathrm{P}=U\left(p^{-1}\right), \quad \mathrm{P} U(k) \mathrm{P}=U(k)
$$

for any $p \in \mathrm{P}$ and $k \in K$. Clearly, this "parity" operator can be viewed as the representative of the Cartan involution $\vartheta(35)$, and its spectrum is $\{ \pm 1\}$. Furthermore, we suppose that its trace can be defined as finite, and not zero, as it has been done for (28). Let $a \mapsto w(a)$ be a function on $A$ which is left and right $K$-invariant (it can be complex-valued). Suppose that this function allows us to define 


$$
\mathrm{M}^{w}:=\frac{1}{\operatorname{tr}(\mathrm{P})} \int_{P \sim G / K} \mathrm{~d} \mu_{P}(p) \mathrm{w}(a(p)) U(p) \mathrm{P}
$$

as a bounded operator (in a weak sense). Here, $a(p)$ is defined from the Cartan factorization (36). The latter means that for any $p \in P$, there exist $k \in K$ and $a \in A \subset P$ such that $p=k a(p) k^{-1}$. With the above assumptions, let us prove that (29) holds as well here, i.e., $\operatorname{tr}(U(p) \mathrm{P})$ does not depend on $p$,

$$
\operatorname{tr}(U(p) \mathrm{P})=\operatorname{tr}(\mathrm{P})
$$

By using the decomposition $p=k a k^{-1}$ from (36), we have

$$
\operatorname{tr}(U(p) \mathrm{P})=\operatorname{tr}\left(U\left(k a k^{-1}\right) \mathrm{P}\right)=\operatorname{tr}\left(U(k) U(a) U\left(k^{-1}\right) \mathrm{P}\right)=\operatorname{tr}\left(U(k) U(a) \mathrm{P} U\left(k^{-1}\right)\right)=\operatorname{tr}(U(a) \mathrm{P}) .
$$

Now, for any element $a$ in the abelian Cartan subalgebra $A$, there exists $a^{\prime} \in A$ such that $a^{\prime 2}=a^{-1}$. Hence, we have

$$
\operatorname{tr}(U(a) \mathrm{P})=\operatorname{tr}\left(U\left(a^{\prime}\right) U(a) \mathrm{P} U\left(a^{\prime-1}\right)\right)=\operatorname{tr}\left(U\left(a^{\prime}\right) U(a) U\left(a^{\prime}\right) \mathrm{P}\right)=\operatorname{tr}(\mathrm{P}) .
$$

Choosing the weight $w$ such that

$$
\int_{P} \mathrm{~d} \mu_{P}(p) w(a(p))=1
$$

there results that $M^{w}$ is a unit trace operator, i.e., $\operatorname{tr} M^{w}=1$.

Introducing its displaced version under the action of $U$,

$$
M^{w}(g)=U(g) M^{w} U^{\dagger}(g)
$$

and supposing that the Haar measure on $K$ is normalized, one derives from (33) the resolution of the identity

$$
\int_{P} \frac{\mathrm{d} \mu_{P}(p)}{C^{w}} \mathrm{M}^{w}(p)=I, \quad C^{w}=\int_{P} \mathrm{~d} \mu_{P}(p) \operatorname{tr}\left(\rho_{0} \mathrm{M}^{w}(p)\right),
$$

where the density operator $\rho_{0}$ has been suitably chosen.

Effectively, starting from (33) and from the integral representation of $\mathrm{M}^{w}$,

$$
\begin{aligned}
I & =\int_{G} \frac{\mathrm{d}_{\text {haar }}(g)}{C^{w}} M^{w}(g) \\
& =\int_{P} \frac{\mathrm{d} \mu_{P}(p)}{C^{w}} \int_{K} \mathrm{~d}_{\text {haar }}(k) \int_{P} \mathrm{~d} \mu_{P}\left(p^{\prime}\right) w\left(a\left(p^{\prime}\right)\right) U(p k) U\left(p^{\prime}\right) U^{\dagger}(p k) \\
& =\int_{P} \frac{\mathrm{d} \mu_{P}(p)}{C^{w}} \int_{K} \mathrm{~d}_{\text {haar }}(k) \int_{P} \mathrm{~d} \mu_{P}\left(p^{\prime}\right) w\left(a\left(p^{\prime}\right)\right) U(p) U\left(k p^{\prime} k^{-1}\right) U^{\dagger}(p) \\
& =\int_{P} \frac{\mathrm{d} \mu_{P}(p)}{C^{w}} \int_{K} \mathrm{~d}_{\text {haar }}(k) \int_{P} \mathrm{~d} \mu_{P}\left(k^{-1} p^{\prime} k\right) w\left(a\left(k^{-1} p^{\prime} k\right)\right) U(p) U\left(p^{\prime}\right) U^{\dagger}(p) \\
& =\int_{P} \frac{\mathrm{d} \mu_{P}(p)}{C^{w}} \int_{P} \mathrm{~d} \mu_{P}\left(p^{\prime}\right) w\left(a\left(p^{\prime}\right)\right) U(p) U\left(p^{\prime}\right) U^{\dagger}(p) \\
& =\int_{P} \frac{\mathrm{d} \mu_{P}(p)}{C^{w}} M^{w}(p) .
\end{aligned}
$$

The integral quantization of functions (or distributions) results from (39),

$$
f(p) \mapsto A_{f}=\int_{P} \frac{\mathrm{d} \mu_{P}(p)}{C^{w}} f(p) M^{w}(p)
$$

\section{SU $(1,1)$ QUANTIZER OPERATOR FROM WEIGHT ON THE UNIT DISK}

\section{A. Construction from weight}

The above material is now implemented in the $\mathrm{SU}(1,1)$ case. Let us pick an $\eta>1 / 2$ and choose a function $[0,1] \ni u \equiv|z|^{2} \mapsto w(u)$ such that the operator,

$$
\mathrm{M}^{w ; \eta}=2 \int_{\mathscr{D}} \frac{\mathrm{d}^{2} z}{\left(1-|z|^{2}\right)^{2}} w\left(|z|^{2}\right) U^{\eta}(p(z)) \mathrm{P}
$$


is bounded and traceclass with unit trace,

$$
\operatorname{tr} M^{w ; \eta}=1
$$

Here, the parity operator $\mathrm{P}$ was defined in (28) and it was been introduced in the general construction (38) by convenience, as its importance will appear soon.

Supposing that we can invert infinite sum and integral, this condition together with (29) imply the following normalization of the weight function $w$,

$$
\int_{\mathscr{D}} \frac{\mathrm{d}^{2} z}{\left(1-|z|^{2}\right)^{2}} w\left(|z|^{2}\right)=1
$$

Thus, if $w$ is non-negative, it can be viewed as a probability distribution on the unit disk equipped with its $\operatorname{SU}(1,1)$ invariant measure $\frac{\mathrm{d}^{2} z}{\left(1-|z|^{2}\right)^{2}}$. From $\left(U^{\eta}(p(z))\right)^{\dagger}=U^{\eta}(p(-z))$ and the invariance of the measure and $w$ under the change $z \mapsto-z$, one infers that $\mathrm{M}^{w ; \eta}$ is symmetric and so self-adjoint. Due to the isotropy of the weight function, $\mathrm{M}^{w ; \eta}$ is diagonal in the basis $\left\{e_{n}\right\}$, with matrix elements deduced from (24) after the change $|z| \mapsto v=1-2|z|^{2}$,

$$
\mathrm{M}_{n n^{\prime}}^{w ; \eta}=\delta_{n n^{\prime}}(-1)^{n} 2^{2-\eta} \pi \int_{-1}^{1} \mathrm{~d} v w\left(\frac{1-v}{2}\right)(1+v)^{\eta-2} P_{n}^{(0,2 \eta-1)}(v)
$$

\section{B. Resolution of the identity}

The unitarily transported versions

$$
M^{w ; \eta}(p(z))=U^{\eta}\left(p(z) M^{w ; \eta} U^{\eta}(p(-z))\right.
$$

of this operator are also bounded self-adjoint and are expected to resolve the unity with respect to a measure on $\mathscr{D}$ proportional to $\mathrm{d}^{2} z /\left(1-|z|^{2}\right)^{2}$

$$
I=\frac{1}{C^{w}} \int_{\mathscr{D}} \frac{\mathrm{d}^{2} z}{\left(1-|z|^{2}\right)^{2}} \mathrm{M}^{w ; \eta}(p(z)) .
$$

One then computes $C^{w}$ with the simplest $\rho_{0}=\left|e_{0}\right\rangle\left\langle e_{0}\right|$,

$$
\begin{aligned}
C^{w} & =\int_{\mathscr{D}} \frac{\mathrm{d}^{2} z}{\left(1-|z|^{2}\right)^{2}}\left\langle e_{0}\left|\mathrm{M}^{w ; \eta}(p(z))\right| e_{0}\right\rangle=\sum_{n} \mathrm{M}_{n n}^{w ; \eta} \int_{\mathscr{D}} \frac{\mathrm{d}^{2} z}{\left(1-|z|^{2}\right)^{2}} U_{0 n}^{\eta}(p(z)) \overline{U_{0 n}^{\eta}(p(z))} \\
& =\sum_{n} \mathrm{M}_{n n}^{w ; \eta} \frac{\Gamma(2 \eta+n)}{n ! \Gamma(2 \eta)} \int_{\mathscr{D}} \mathrm{d}^{2} z\left(1-|z|^{2}\right)^{2 \eta-2}|z|^{2 n} \\
& =\sum_{n} \mathrm{M}_{n n}^{w ; \eta} \frac{\Gamma(2 \eta+n)}{n ! \Gamma(2 \eta)} \int_{0}^{2 \pi} \int_{0}^{1} r^{2 n+1}\left(1-r^{2}\right)^{2 \eta-2} \mathrm{~d} \theta \mathrm{d} r \\
& =\sum_{n} \mathrm{M}_{n n}^{w ; \eta} \frac{\Gamma(2 \eta+n)}{n ! \Gamma(2 \eta)} 2 \pi \int_{0}^{1} x^{n}(1-x)^{2 \eta-2} \mathrm{~d} x \\
& =\frac{\pi}{2 \eta-1} \sum_{n} \mathrm{M}_{n n}^{w ; \eta}=\frac{\pi}{2 \eta-1},
\end{aligned}
$$

where we have used the expression (22) for $U_{0 n}^{\eta}(p(z))$, performed the variable changes $z=r e^{\mathrm{i} \theta}$ (such that $\left.\mathrm{d} z^{2}=r \mathrm{~d} r \mathrm{~d} \theta\right)$ and next $r^{2}=x$, taken in consideration the integral representation of the beta function,

$$
\beta(a, b)=\int_{0}^{1} x^{a-1}(1-x)^{b-1} \mathrm{~d} x=\frac{\Gamma(a) \Gamma(b)}{\Gamma(a+b)},
$$

and applied the unit trace of $\mathrm{M}^{w ; \eta}$. Therefore, the resolution of the identity holds with the measure

$$
I=\frac{2 \eta-1}{\pi} \int_{\mathscr{D}} \frac{\mathrm{d}^{2} z}{\left(1-|z|^{2}\right)^{2}} \mathrm{M}^{w ; \eta}(p(z)) .
$$

\section{Particular weight functions}

Let us consider the particular family of positive weight functions,

$$
w_{s}(u):=\frac{s-1}{\pi}(1-u)^{s}, \quad s>1,
$$


which satisfy (41). Using (A2), we have for the matrix elements of $M^{w_{s} ; \eta}$,

$$
\begin{aligned}
\mathrm{M}_{n n^{\prime}}^{w_{s} ; \eta} & =(-1)^{n} \delta_{n n^{\prime}} 2(s-1) \frac{\Gamma(\eta+s-1) \Gamma(s-\eta)}{\Gamma(\eta+s+n) \Gamma(s-\eta-n)} \\
& = \begin{cases}\delta_{n n^{\prime}} 2(s-1) \frac{\Gamma(\eta+s-1) \Gamma(\eta-s+n+1)}{\Gamma(\eta+s+n) \Gamma(\eta-s+1)} & \text { for } s \neq \eta+1, \\
\delta_{n n^{\prime}} \delta_{n 0} & \text { for } s=\eta+1 .\end{cases}
\end{aligned}
$$

From these expressions, we see that the operator $\mathrm{M}^{w_{s} ; \eta}$ is a density operator if $1<s \leq \eta+1$. Positiveness is lost for $s>\eta+1$. It is a finite rank $=p$ operator for all $s=\eta+p, p=1,2, \ldots$. The limit case $s=\eta+1$ in (45) corresponds to Perelomov SU $(1,1)$ coherent states ${ }^{13}$ (with $\bar{z}$ instead of $z$ ),

$$
\mathrm{M}^{w_{\eta+1} ; \eta}=\left|e_{0}\right\rangle\left\langle e_{0}\left|, \quad \mathrm{M}^{w_{\eta+1} ; \eta}(p(\bar{z}))=\right| z ; \eta\right\rangle\langle z ; \eta|,
$$

with

$$
|z ; \eta\rangle=\left(1-|z|^{2}\right)^{\eta} \sum_{n=0}^{\infty} \sqrt{\frac{(2 \eta)_{n}}{n !}} z^{n}\left|e_{n}\right\rangle=U^{\eta}(p(\bar{z}))\left|e_{0}\right\rangle .
$$

See also Chap. 8 in Ref. 29 for more details and specific properties.

It is interesting to determine the weight function $w_{e_{m} ; \eta}$ yielding the projector $\left|e_{m}\right\rangle\left\langle e_{m}\right|$ through (40). By using the orthogonality relations satisfied by the Jacobi polynomials, we find

$$
\begin{aligned}
\left|e_{m}\right\rangle\left\langle e_{m}\right| & =2 \int_{\mathscr{D}} \frac{\mathrm{d}^{2} z}{\left(1-|z|^{2}\right)^{2}} w_{e_{m} ; \eta}\left(|z|^{2}\right) U^{\eta}(p(z)) \mathrm{P}, \\
w_{e_{m} ; \eta}\left(|z|^{2}\right) & =(-1)^{m} \frac{\eta+m}{\pi}\left(1-|z|^{2}\right)^{\eta+1} P_{m}^{(0,2 \eta-1)}\left(1-2|z|^{2}\right) .
\end{aligned}
$$

Clearly, at the exception of $m=0$, the weights $w_{e_{m} ; \eta}$ are not non-negative.

It is naturally possible to extend the range of values of $s$ below the limit 1 at the price to violate integrability and positivity of the weight function. As a matter of fact, there exists a remarkable value of $s$, namely, $s=1 / 2$, for which the suitably renormalized weight function

$$
w(u)=\frac{2 \eta-1}{4 \pi}(1-u)^{1 / 2}
$$

yields the identity operator,

$$
I=\mathrm{M}^{w_{1 / 2} ; \eta}=\frac{2 \eta-1}{2 \pi} \int_{\mathscr{D}} \frac{\mathrm{d}^{2} z}{\left(1-|z|^{2}\right)^{3 / 2}} U^{\eta}(p(z)) \mathrm{P} .
$$

From the above, we obtain the integral representation of unit trace twice the parity operator,

$$
2 \mathrm{P}=\frac{2 \eta-1}{\pi} \int_{\mathscr{D}} \frac{\mathrm{d}^{2} z}{\left(1-|z|^{2}\right)^{3 / 2}} U^{\eta}(p(z)) .
$$

As an interesting consequence of (48) combined with the resolution of the identity (43) and

$$
\left.U^{\eta}(p(z)) \mathrm{P} U^{\eta}(p(-z))=U^{\eta}(p(z))^{2}\right) \mathrm{P}=U^{\eta}\left(p\left(\frac{2 z}{1+|z|^{2}}\right)\right) \mathrm{P},
$$

we obtain

$$
I=2 \frac{2 \eta-1}{\pi} \int_{\mathscr{D}} \frac{\mathrm{d}^{2} z}{\left(1-|z|^{2}\right)^{2}} U^{\eta}\left(p\left(\frac{2 z}{1+|z|^{2}}\right)\right) \mathrm{P} .
$$

There results the (non-trivial) integral formula given in (A) for hypergeometric polynomials.

Remark. An open question is to establish an inverse formula allowing to rebuild the weight $w$ from some trace formula as it exists for the Weyl-Heisenberg or the affine case (see, for instance, Refs. 31 and 32). More precisely, given $w$, the problem is to determine an operator $\mathfrak{I}$ such that the following reconstruction formula holds:

$$
w\left(|z|^{2}\right)=\operatorname{tr}\left(M^{w ; \eta} U^{\eta}(p(-z) \mathfrak{I}) .\right.
$$




\section{WEIGHTED SU( 1,1$)$ INTEGRAL QUANTIZATIONS FOR THE UNIT DISK}

We now start from the framework of Sec. $V$ and establish general formulas for the quantization issued from a weight function $w(u)$ yielding the operator $\mathrm{M}^{w ; \eta}$ in $(40)$,

$$
f \mapsto \mathrm{A}_{f}^{w ; \eta}=\frac{2 \eta-1}{\pi} \int_{\mathscr{D}} \frac{\mathrm{d}^{2} z}{\left(1-|z|^{2}\right)^{2}} f(z) \mathrm{M}^{w ; \eta}(p(z)) .
$$

Since the operator $A_{f}^{w ; \eta}$ acts on the Fock-Bargmann Hilbert space, the most straightforward way to characterize it is to compute its matrix elements with respect to the orthonormal basis (19). We know from (42) that the operator $M^{w ; \eta}$ is diagonal. Hence, the general form of those matrix elements reads as

$$
\begin{aligned}
\left(\mathrm{A}_{f}^{w ; \eta}\right)_{n n^{\prime}} & =\frac{2 \eta-1}{\pi} \int_{\mathscr{D}} \frac{\mathrm{d}^{2} z}{\left(1-|z|^{2}\right)^{2}} f(z) \mathrm{M}^{w ; \eta}(p(z))_{n n^{\prime}} \\
& =\frac{2 \eta-1}{\pi} \sum_{k} \mathrm{M}_{k k}^{w ; \eta} \int_{\mathscr{D}} \frac{\mathrm{d}^{2} z}{\left(1-|z|^{2}\right)^{2}} f(z) U_{n k}^{\eta}(p(z)) \overline{U_{n^{\prime} k}^{\eta}(p(z))} \\
& :=\frac{2 \eta-1}{\pi} \sum_{k} \mathrm{M}_{k k}^{w ; \eta} \mathscr{I}_{k, n, n^{\prime}}^{\eta}(f) .
\end{aligned}
$$

The integral in the lhs has the explicit form derived from (22),

$$
\begin{aligned}
\mathscr{I}_{k, n, n^{\prime}}^{\eta}(f)= & \int_{\mathscr{D}} \frac{\mathrm{d}^{2} z}{\left(1-|z|^{2}\right)^{2}} f(z) U_{n k}^{\eta}(p(z)) \overline{U_{n^{\prime} k}^{\eta}(p(z))} \\
= & \left(\frac{n_{<} ! \Gamma\left(2 \eta+n_{>}\right)}{n>! \Gamma\left(2 \eta+n_{<}\right)}\right)^{1 / 2}\left(\frac{n_{<}^{\prime} ! \Gamma\left(2 \eta+n_{>}^{\prime}\right)}{n_{>}^{\prime} ! \Gamma\left(2 \eta+n_{<}^{\prime}\right)}\right)^{1 / 2}(\operatorname{sgn}(n-k))^{n-k}\left(\operatorname{sgn}\left(n^{\prime}-k\right)\right)^{n^{\prime}-k}, \\
& \quad \times \int_{\mathscr{D}} \mathrm{d}^{2} z f(z)\left(1-|z|^{2}\right)^{2 \eta-2}|z|^{n_{>}-n_{<}+n_{>}^{\prime}-n_{<}^{\prime}} e^{\mathrm{i}\left(n^{\prime}-n\right) \phi} \\
& \quad \times P_{n_{<}}^{\left(n_{>}-n_{<}, 2 \eta-1\right)}\left(1-2|z|^{2}\right) P_{n_{<}^{\prime}}^{\left(n_{>}^{\prime}-n_{<}^{\prime}, 2 \eta-1\right)}\left(1-2|z|^{2}\right),
\end{aligned}
$$

with

$$
\underset{<>}{n>}=\left\{\begin{array}{l}
\max \\
\min
\end{array}(n, k), \quad \underset{n_{>}^{\prime}}{\prime}=\left\{\begin{array}{l}
\max \\
\min
\end{array}\left(n^{\prime}, k\right) .\right.\right.
$$

In the isotropic case $f(z)=l\left(|z|^{2}\right)$, this integral simplifies to

$$
\begin{aligned}
\mathscr{I}_{k, n, n^{\prime}}^{\eta}(f)=\pi \delta_{n n^{\prime}} & 2^{1-2 \eta+n_{<}-n_{>}} \frac{n_{<} ! \Gamma\left(2 \eta+n_{>}\right)}{n_{>} ! \Gamma\left(2 \eta+n_{<}\right)} \\
& \times \int_{-1}^{+1} \mathrm{~d} v l\left(\frac{1-v}{2}\right)(1-v)^{n_{>}-n_{<}}(1+v)^{2 \eta-2}\left(P_{n_{<}}^{\left(n_{>}-n_{<}, 2 \eta-1\right)}(v)\right)^{2},
\end{aligned}
$$

where we have used the variable $v=1-2|z|^{2}$. Actually, it is sufficient to consider the case $n \leq k$, for which

$$
\begin{aligned}
\mathscr{I}_{k, n, n^{\prime}}^{\eta}(f)=\pi \delta_{n n^{\prime}} & 2^{1-2 \eta+n-k} \frac{n ! \Gamma(2 \eta+k)}{k ! \Gamma(2 \eta+n)} \\
& \times \int_{-1}^{+1} \mathrm{~d} v l\left(\frac{1-v}{2}\right)(1-v)^{k-n}(1+v)^{2 \eta-2}\left(P_{n}^{(k-n, 2 \eta-1)}(v)\right)^{2},
\end{aligned}
$$

the case $k<n$ keeping the same form thanks to the formula for Jacobi polynomials, ${ }^{26}$

$$
P_{n}^{(-a, \beta)}(x)=\frac{\Gamma(n+\beta+1)(n-a) !}{\Gamma(n+\beta+1-a) n !}\left(\frac{x-1}{2}\right) P_{n-a}^{(a, \beta)}(x) \quad \text { for } \quad a \in \mathbb{N} .
$$

Of course, for $f=1=l$, we should recover the identity in (50), which implies the following value for the integral $\mathscr{I}_{k, n, n^{\prime}}^{\eta}(1)$ (which can be found in Ref. 30),

$$
\begin{aligned}
\mathscr{I}_{k, n, n^{\prime}}^{\eta}(1) & =\pi \delta_{n n^{\prime}} 2^{1-2 \eta+n-k} \frac{n ! \Gamma(2 \eta+k)}{k ! \Gamma(2 \eta+n)} \int_{-1}^{+1} \mathrm{~d} v(1-v)^{k-n}(1+v)^{2 \eta-2}\left(P_{n}^{(k-n, 2 \eta-1)}(v)\right)^{2} \\
& =\frac{\pi}{2 \eta-1} .
\end{aligned}
$$


Another useful particular case is $f\left(|z|^{2}\right)=\left(1-|z|^{2}\right)^{-1}=2 /(1+v)$. Then,

$$
\begin{aligned}
\mathscr{I}_{k, n, n^{\prime}}^{\eta}\left(\frac{1}{1-|z|^{2}}\right) & =\pi \delta_{n n^{\prime}} 2^{2-2 \eta+n-k} \frac{n ! \Gamma(2 \eta+k)}{k ! \Gamma(2 \eta+n)} \int_{-1}^{+1} \mathrm{~d} v(1-v)^{k-n}(1+v)^{2 \eta-3}\left(P_{n}^{(k-n, 2 \eta-1)}(v)\right)^{2} \\
& =\delta_{n n^{\prime}} \frac{\pi}{2 \eta-1} \frac{1}{2 \eta(\eta-1)}[(k+\eta)(\eta+n)+\eta(\eta-1)] .
\end{aligned}
$$

This formula is derived from Eq. (A1).

By construction, the quantization map (49) is covariant with respect to the unitary action $U^{\eta}$ of $S U(1,1)$,

$$
U^{\eta}\left(g_{0}\right) A_{f}^{w ; \eta} U^{\eta \dagger}\left(g_{0}\right)=A_{\mathfrak{U}\left(g_{0}\right) f}^{w ;},
$$

where we recall that $(\mathfrak{U}(g) f)(z)=f\left(g^{-1} \cdot z\right)$. Moreover, due to the self-adjointness of $\mathrm{M}^{w ; \eta}(p(z))$, we have the relation

$$
\left(\mathrm{A}_{f}^{w ; \eta}\right)^{\dagger}=\mathrm{A}_{\bar{f}}^{w ; \eta}
$$

The following important statement results from the covariance (53) and self-adjointness property (54).

Proposition VI.1 Suppose that $w$ and $\eta>1 / 2$ are such that the series

$$
\mathscr{S}^{w ; \eta}:=\sum_{k=0}^{\infty} k \mathrm{M}_{k k}^{w ; \eta}
$$

converges in a certain sense. Then, the quantization (49) maps the basic observables $k_{a}, a=0,1,2$ (resp. $a=0, \pm$ ), defined in (10), to the selfadjoint generators (31) up to a constant factor $\gamma_{w ; \eta}$,

$$
\mathrm{A}_{k_{a}}^{w ; \eta}=\gamma_{w ; \eta} K_{a}, \quad a=0,1,2, \quad \text { resp. } a=0, \pm,
$$

with

$$
\gamma_{w ; \eta}=\frac{1}{\eta-1}\left[1+\frac{\mathscr{S}^{w ; \eta}}{\eta}\right]
$$

Proof. We have from (49),

$$
A_{k_{a}}=\frac{2 \eta-1}{\pi} \int_{\mathscr{D}} \frac{\mathrm{d}^{2} z}{\left(1-|z|^{2}\right)^{2}} k_{a}(z) \mathrm{M}^{w ; \eta}(p(z)) .
$$

From the covariance (53) and from (15),

$$
U^{\eta}(g) A_{k_{a}}^{w ; \eta} U^{\eta \dagger}(g)=A_{\mathscr{U}(g) k_{a}}^{w ; \eta}=\sum_{b}[\mathfrak{U}(g)]_{b a} A_{k_{b}}^{w ; \eta}, \quad \forall g \in \mathrm{SU}(1,1) .
$$

This means that the operators $\mathrm{A}_{k_{a}}^{w ; \eta}$ transform under the action of $U^{\eta}(g)$ exactly like the operators $K_{a}$. Thus, there exists a constant $\gamma_{w ; \eta}$ depending on $\eta$ and $w$ such that

$$
\mathrm{A}_{k_{a}}^{w ; \eta}=\gamma_{w ; \eta} K_{a} .
$$

The constant is calculated by picking $a=0$ and considering the lowest matrix elements via (52),

$$
\begin{aligned}
\gamma_{w ; \eta}\left(K_{0}\right)_{00} & =\gamma_{w ; \eta} \eta=\left(\mathrm{A}_{k_{0}}^{w ; \eta}\right)_{00}=\frac{2 \eta-1}{\pi} \sum_{k} \mathrm{M}_{k k}^{w ; \eta} \mathscr{I}_{k, 0,0}^{\eta}\left(k_{0}\right) \\
& =2 \frac{2 \eta-1}{\pi} \sum_{k} \mathrm{M}_{k k}^{w ; \eta} \mathscr{I}_{k, 0,0}^{\eta}\left(\frac{1}{\left(1-|z|^{2}\right.}\right)-1 \\
& =\frac{1}{\eta-1}\left[\eta+\mathscr{S}^{w ; \eta}\right],
\end{aligned}
$$

we finally get (56).

Once it is proved for $A_{k_{0}}^{w ; \eta}$, it is also proved for $A_{k_{ \pm}}^{w ; \eta}$ by using (57) with specific elements $g^{\text {'s }}$ mapping $K_{0}$ to $K_{ \pm}$. 
For instance, if we choose $w=w_{s}$ given by (44) with $s=\eta+1$ (Perelomov coherent states case), formula (45) gives $\mathscr{S}^{w_{\eta+1} ; \eta}=0$ and so

$$
\gamma_{w_{\eta+1} ; \eta}=\frac{1}{\eta-1}
$$

We note that with the particular value $\eta=2$, the quantization of basic observables is exact. In the case of coherent states built from $\left|e_{m}\right\rangle$ and giving rise to (47), the constant $\gamma_{w ; \eta}$ is given by

$$
\gamma_{w_{e_{m}} ; \eta}=\frac{\eta+m}{\eta(\eta-1)}
$$

Another interesting case related to $w=w_{s}$ concerns the already mentioned limit value $s=1 / 2$ for which $\mathscr{S}^{w_{1 / 2} ; \eta}=2 \sum_{k=0}^{\infty} k(-1)^{k}=-1 / 2$ (in Abel sense). Then,

$$
\gamma_{w_{1 / 2} ; \eta}=\frac{2 \eta-1}{2 \eta(\eta-1)}
$$

and there is no real value of $\eta$ for which the quantization of the basic observables is exact.

Finally, the interesting functions to be quantized have the general form $f(z)=h\left(|z|^{2}\right) z^{a}, a \in \mathbb{N}$, where $h$ is real-valued. Note that the quantization of the conjugate is straightforward, due to the relation (54). However, in view of the technicality of the calculations, we will not pursue in this way.

\section{QUANTUM PHASE SPACE PORTRAITS}

Let us consider a weight function $w\left(|z|^{2}\right)$ yielding the symmetric unit trace operator $M^{w ; \eta}$ through Eq. (40). The lower symbol or quantum phase space portrait of an operator $A$ in $\mathscr{H}$ is the function,

$$
\check{A}(z):=\operatorname{tr}\left(A U^{\eta}(p(z)) M^{w ; \eta}\left(U^{\eta}(p(z))^{\dagger}\right)=\operatorname{tr}\left(A M^{w ; \eta}(p(z))\right) .\right.
$$

Let us now consider a function $f(z)$ and its quantum version $\mathrm{A}_{f}^{w_{1} ; \eta}$ built from a first weight function $w_{1}\left(|z|^{2}\right)$, used for the "analysis." Its lower symbol associated with a second weight function $w_{2}\left(|z|^{2}\right)$, used for the "reconstruction" (terms borrowed from signal analysis terminology) reads as the map

$$
\begin{aligned}
f(z) \mapsto \check{f}(z) & \equiv \check{\mathrm{A}}_{f}^{w_{12} ; \eta}(z) \\
& =\frac{2 \eta-1}{\pi} \int_{\mathscr{D}} \frac{\mathrm{d}^{2} z^{\prime}}{\left(1-\left|z^{\prime}\right|^{2}\right)^{2}} f\left(z^{\prime}\right) \operatorname{tr}\left(\mathrm{M}^{w_{1} ; \eta}\left(p(-z) p\left(z^{\prime}\right)\right) \mathrm{M}^{w_{2} ; \eta}\right) .
\end{aligned}
$$

Now, we have the SU $(1,1)$ composition formula,

$$
p(-z) p\left(z^{\prime}\right)=p(t) h(\theta), \quad \text { with } t=p(-z) \cdot z^{\prime}, \quad h(\theta) \in H .
$$

Since $U^{\eta}(h(\theta))$ is diagonal, it commutes with $\mathrm{M}^{w ; \eta}$, and we obtain after the change of variable $z^{\prime} \mapsto t$,

$$
\check{f}(z)=\frac{2 \eta-1}{\pi} \int_{\mathscr{D}} \frac{\mathrm{d}^{2} t}{\left(1-|t|^{2}\right)^{2}} f(p(z) \cdot t) \operatorname{tr}\left(\mathrm{M}^{w_{1} ; \eta}(p(t)) \mathrm{M}^{w_{2} ; \eta}\right) .
$$

Clearly, since for $f=1$ the rhs is equal to 1 , the map

$$
t \mapsto \operatorname{tr}\left(\mathrm{M}^{w_{1} ; \eta}(p(t)) \mathrm{M}^{w_{2} ; \eta}\right)=\operatorname{tr}\left(U^{\eta}(p(t)) \mathrm{M}^{w_{1} ; \eta}\left[\mathrm{M}^{w_{2} ; \eta} U^{\eta}(p(t))\right]^{\dagger}\right)
$$

is a probability distribution on the unit disk $\mathscr{D}$ with respect to the measure $\frac{2 \eta-1}{\pi} \frac{\mathrm{d}^{2} t}{\left(1-|t|^{2}\right)^{2}}$ if $\mathrm{M}^{w_{1} ; \eta}$ and $\mathrm{M}^{w_{2} ; \eta}$ are nonnegative, i.e., are density operator.

Let us just prove that the lower symbols of the three generators $K_{0}, K_{ \pm}$, are proportional to their classical counterpart,

$$
\check{\mathrm{A}}_{k_{i}}^{w_{12} ; \eta}(z)=\varkappa_{w_{12} ; \eta} k_{i}(z), \quad i=0,1,2 .
$$

This proof is similar to the one of Proposition VI.1. Let us apply the regular representation of SU $(1,1)$ on both sides of Eq. (60), 


$$
\check{f}\left(g^{-1} \cdot z\right)=\frac{2 \eta-1}{\pi} \int_{\mathscr{D}} \frac{\mathrm{d}^{2} t}{\left(1-|t|^{2}\right)^{2}} f\left(p\left(g^{-1} \cdot z\right) \cdot t\right) \operatorname{tr}\left(\mathrm{M}^{w_{1} ; \eta}(p(t)) \mathrm{M}^{w_{2} ; \eta}\right) .
$$

We now apply (7),

$$
p\left(g^{-1} \cdot z\right) \cdot t=\left(g^{-1} p(z) h\right) \cdot t=g^{-1} \cdot(p(z) \cdot(h \cdot t)), \quad \text { with } h \in \mathrm{U}(1) .
$$

After changing $h . t \mapsto t$ and using the invariance or the measure and of the trace, we get

$$
\check{f}\left(g^{-1} \cdot z\right)=\frac{2 \eta-1}{\pi} \int_{\mathscr{D}} \frac{\mathrm{d}^{2} t}{\left(1-|t|^{2}\right)^{2}} f\left(g^{-1} \cdot(p(z) \cdot t)\right) \operatorname{tr}\left(\mathrm{M}^{w_{1} ; \eta}(p(t)) \mathrm{M}^{w_{2} ; \eta}\right) .
$$

Hence, by particularizing to $f=k_{a}, a=0, \pm$, we check by linearity that their corresponding $\hat{k}_{a}$ transform exactly in the same way as in (12), under the regular representation of $\mathrm{SU}(1,1)$. This proves the proportionality relation (61). The constant is computed by using a similar trick to (58). Of course, these formulas are valid only if $\eta$ and the weights $w_{1}, w_{2}$ are such that the integral (60) converges for each one of the considered cases. As an elementary example, let us choose $w_{1}(u)=w_{2}(u)=(\eta / \pi)(1-u)^{\eta+1}$, which corresponds to the Perelomov case (46). Using the transformations (16), we find for (60),

$$
\begin{aligned}
\check{f}_{a}(z) & =\frac{2 \eta-1}{\pi} \int_{\mathscr{D}} \frac{\mathrm{d}^{2} t}{\left(1-|t|^{2}\right)^{2}} k_{a}\left((p(z) \cdot t)|\langle t ; \eta \mid 0 ; \eta\rangle|^{2}\right. \\
& =\frac{2 \eta_{1}-1}{\pi} \int_{\mathscr{D}} \mathrm{d}^{2} t\left(1-|t|^{2}\right)^{2 \eta-2} k_{a}((p(z) \cdot t) \\
& =k_{a}(z)\left[\frac{2 \eta-1}{\pi} \int_{\mathscr{D}} \mathrm{d}^{2} t\left(1-|t|^{2}\right)^{2 \eta-2} k_{0}(t)\right] \\
& =\frac{2 \eta}{\eta-1} k_{a}(z) .
\end{aligned}
$$

Note that this proportionality coefficient cannot be put equal to 1 for the allowed range $\eta>1$. An interesting problem is to choose $\eta=2, w_{1}(u)$ $=(\eta / \pi)(1-u)^{\eta+1}=2 \pi(1-u)^{3}$ which corresponds to a Perelomov case and which yields the exact quantization for the three basic observables, and to build the reconstruction operator $\mathrm{M}^{w_{2} ; \eta}$ which yields exactly $\check{f}_{a}(z)=k_{a}(z)$.

\section{CONCLUSION}

Given a semi-simple group $G$, we have generalized the covariant integral quantization of functions defined on the coset $G / K$ by introducing a weight function combined with the parity operator associated with the Cartan involution. According to the choice of such weight functions, we obtain different quantizations. We have implemented this procedure in the case of the group SU $(1,1)$. Given an irreducible unitary representation $U^{\eta}, \eta>1$ in the discrete series of $S U(1,1)$, we have presented a family of covariant integral quantizations of functions on the open unit disk. A physical interpretation is to consider $\mathrm{SU}(1,1)$ as the kinematical group of the $1+1$ AdS space-time and the unit disk as the phase space for the motion of a "massive" Wigner elementary system in AdS. In this example, each quantization is determined by an isotropic weight function on the disk or equivalently by a unit trace operator viewed as an " $U$ " -Fourier transform" of this weight. Perelomov coherent states quantizations are particular cases. Reversal of these quantizations under the form of semi-classical portraits of quantized versions of a classical object has been defined as local averaging of the latter, involving a second weight function. In this regard, a non-trivial question to be considered is to determine a pair $\left(w_{1}, w_{2}\right)$ of weight functions for which the reversal is exact in the Wigner-Weyl sense, i.e., the following holds:

$$
f(z) \mapsto \check{f}(z) \equiv \check{\mathrm{A}}_{f}^{w_{12} ; \eta}(z)=f(z),
$$

with the notations of (59). To a certain extent, this problem could be viewed as a generalization to $\mathrm{SU}(1,1)$ of similar approaches concerning the Weyl-Heisenberg group, the affine group, and more general groups, and their related Weyl operators and Wigner functions defined in a wide sense; see, for instance, Refs. 31-36 and reference therein. Finally, appealing generalizations of the presented material can be considered for the higher-dimensional anti-de Sitter groups, particularly $\mathrm{SO}(2,3), 5$ since some of these groups might have physically relevant discrete series.

\section{ACKNOWLEDGMENTS}

This research was supported in part by the Ministerio de Economía y Competitividad of Spain under Grant No. MTM2014-57129-C21-P and the Junta de Castilla y León (Grant Nos. VA137G18 and BU229P18). J.P.G. is also indebted to the University of Valladolid for its hospitality. 


\section{APPENDIX: USEFUL INTEGRALS WITH JACOBI POLYNOMIALS AND OTHERS}

Orthogonality

$$
\begin{aligned}
\int_{-1}^{+1} \mathrm{~d} v(1-v)^{\alpha}(1+v)^{\beta} P_{m}^{(\alpha, \beta)}(v) P_{n}^{(\alpha, \beta)}(v) & \\
& =\delta_{m n} \frac{2^{\alpha+\beta+1}}{\alpha+\beta+2 n+1} \frac{\Gamma(\alpha+n+1) \Gamma(\beta+n+1)}{n ! \Gamma(\alpha+\beta+n+1)}, \quad \alpha>-1, \beta>-1 .
\end{aligned}
$$

Others

From Gradshteyn-Ryzhik 7.391 in Ref. 30,

$$
\int_{-1}^{+1} \mathrm{~d} v(1-v)^{\alpha}(1+v)^{\beta-1}\left(P_{n}^{(\alpha, \beta)}(v)\right)^{2}=\frac{2^{\alpha+\beta}}{\beta} \frac{\Gamma(\alpha+n+1) \Gamma(\beta+n+1)}{n ! \Gamma(\alpha+\beta+n+1)}, \quad \alpha>-1, \beta>0 .
$$

A new one

$$
\begin{aligned}
\int_{-1}^{+1} \mathrm{~d} v(1-v)^{\alpha}(1+v)^{\beta-2}\left(P_{n}^{(\alpha, \beta)}(v)\right)^{2} \\
\quad=\frac{2^{\alpha+\beta-1}}{\beta(\beta+1)(\beta-1)} \frac{(\Gamma(\alpha+n+1) ! \Gamma(\beta+n+1)}{n ! \Gamma(\alpha+\beta+n+1)}[(\beta+1)(\alpha+\beta)+2(\alpha+\beta+n+1) n],
\end{aligned}
$$

for $\alpha>-1$ and $\beta>1$.

A third formula more

$$
\begin{aligned}
\int_{-1}^{1} \mathrm{~d} x & (1-x)^{\rho}(1+x)^{\sigma} P_{n}^{(\mu, v)}(x) \\
& =2^{\rho+\sigma+1} \frac{\Gamma(\rho+1) \Gamma(\sigma+1) \Gamma(n+1+\mu)}{n ! \Gamma(\rho+\sigma+2) \Gamma(\mu+1)}{ }_{3} F_{2}(-n, \mu+v+n+1, \rho+1 ; \mu+1, \rho+\sigma+2 ; 1),
\end{aligned}
$$

with $\operatorname{Re} \rho>-1, \operatorname{Re} \sigma>-1$.

A new integral for hypergeometric polynomials

$$
2(2 \eta-1) \int_{0}^{1} \mathrm{~d} u(1-u)^{2 \eta-2}(1+u)^{-2 \eta}{ }_{2} F_{1}\left(-n, n+2 \eta ; 1 ; \frac{4 u}{(1+u)^{2}}\right)=(-1)^{n} .
$$

Two integral forms for beta function

$$
\begin{aligned}
\beta(x, y)=\frac{\Gamma(x) \Gamma(y)}{\Gamma(x+y)} & =\int_{0}^{1} \mathrm{~d} t t^{x-1}(1-t)^{y-1} \\
& =2^{1-x-y} \int_{-1}^{1} \mathrm{~d} t(1-t)^{x-1}(1+t)^{y-1}
\end{aligned}
$$

\section{REFERENCES}

${ }^{1}$ J.-P. Gazeau and V. Hussin, “Poincaré contraction of SU(1, 1) Fock-Bargmann structure,” J. Phys. A: Math. Gen. 25, 1549-1573 (1992).

${ }^{2}$ J.-P. Gazeau and J. Renaud, "Lie algorithm for an interacting SU(1, 1) elementary system and its contraction," Ann. Phys. 222, 89-121 (1993); Erratum, 235, 242 (1994).

${ }^{3}$ J.-P. Gazeau and J. Renaud, "Relativistic harmonic oscillator and space curvature," Phys. Lett. A 179, 67-71 (1993).

${ }^{4}$ A. M. Elgradechi and S. De Bièvre, "Phase space quantum mechanics on the anti-de Sitter spacetime and its Poincaré contraction," Ann. Phys. 235, 1-34 (1994).

${ }^{5}$ R. Balbinot, A. El Gradechi, J.-P. Gazeau, and B. Giorgini, “Phase spaces for quantum elementary systems in anti-de Sitter and Minkowski spacetimes,” J. Phys. A: Math. Gen. 25, 1185-1210 (1992).

${ }^{6}$ H. Bergeron and J.-P. Gazeau, "Integral quantizations with two basic examples," Ann. Phys. 344, 43-68 (2014); arXiv:1308.2348 [quant-ph, math-ph].

${ }^{7}$ S. T. Ali, J.-P. Antoine, and J.-P. Gazeau, Coherent States, Wavelets and Their Generalizations, 2nd ed., Theoretical and Mathematical Physics (Springer, New York, 2013).

${ }^{8}$ J.-P. Gazeau, "Covariant integral quantizations and their applications to quantum cosmology," Acta Polytech. 56, 173-179 (2016).

${ }^{9}$ J.-P. Gazeau and R. Murenzi, “Covariant affine integral quantization(s)," J. Math. Phys. 57, 052102-1-052102-22 (2016); arXiv:1512.08274 [quant-ph].

${ }^{10}$ E. H. Lieb, "The classical limit of quantum spin systems," Commun. Math. Phys. 31, 327 (1973).

${ }^{11}$ F. A. Berezin, "Quantization," Math. USSR Izvestija 8, 1109 (1974).

${ }^{12}$ F. A. Berezin, "General concept of quantization,” Commun. Math. Phys. 40, 153 (1975).

${ }^{13}$ A. M. Perelomov, Generalized Coherent States and Their Applications (Springer, Berlin, 1986).

${ }^{14}$ A. A. Kirillov, Lectures on the Orbit Method, Graduate Studies in Mathematics Vol. 64 (American Mathematical Society, Providence, RI, 2004). 
${ }^{15}$ N. M. J. Woodhouse, Geometric Quantization, 2nd ed., Oxford Mathematical Monographs (Oxford Science Publications; The Clarendon Press; Oxford University Press, New York, 1992).

${ }^{16}$ E. Lerman, "Geometric quantization: A crash course," in Mathematical Aspects of Quantization (American Mathematical Society, Provicence, RI, 2012), Vol. 583, pp. 147-174.

${ }^{17}$ F. Bayen, M. Flato, C. Fronsdal, A. Lichnerowicz, and D. Sternheimer, “Deformation theory and quantization. I," Ann. Phys. 111, 61-110 (1978); “Deformation theory and quantization. II," Ann. Phys. 111, 111-151 (1978).

${ }^{18}$ S. Klimek and A. Lesniewski, “Quantum Riemann surfaces I,” Commun. Math. Phys. 146, 103-122 (1992).

${ }^{19}$ S. T. Ali and M. Englis, "Quantization methods: A guide for physicists and analysts," Rev. Math. Phys. 17, 391-490 (2005).

${ }^{20}$ J. H. Rawnsley, "Coherent states and Kähler manifolds," Q. J. Math. Oxford Ser. 28, 403-415 (1977).

${ }^{21}$ J. H. Rawnsley, M. Cahen, and S. Gutt, “Quantization of Kähler manifolds. I. Geometric interpretation of Berezin’s quantization,” J. Geom. Phys. 7, 45-62 (1990).

${ }^{22}$ B. C. Hall, “Berezin-Toeplitz quantization on Lie groups,” J. Funct. Anal. 255, 2488-2506 (2008).

${ }^{23}$ N. J. Vilenkin, Special Functions and the Theory of Group Representations (American Mathematical Society, Providence RI, 1968).

${ }^{24}$ B. Doubrovine, S. Novikov, and A. Fomenko, Géométrie Contemporaine, Mèthodes et Applications I (Mir, Moscow, 1982).

${ }^{25}$ W. Miller, Jr., Lie Theory and Special Functions (Academic, New York, 1968).

${ }^{26}$ W. Magnus, F. Oberhettinger, and R. P. Soni, Formulas and Theorems for the Special Functions of Mathematical Physics (Springer-Verlag, Berlin, 1966).

${ }^{27}$ B. C. Hall, "The range of the heat operator," in The Ubiquitous Heat Kernel, Contemporary Mathematics Vol. 398, edited by J. Jorgenson and L. Walling (American Mathematical Society, Providence, RI, 2006), pp. 203-231.

${ }^{28}$ S. Helgason, Differential Geometry, Lie Groups and Symmetric Spaces (Academic, San Diego, 1978).

${ }^{29}$ J.-P. Gazeau, Coherent States in Quantum Physics (Wiley VCH, Berlin, 2009).

${ }^{30}$ I. S. Gradshteyn and I. M. Ryzhik, in Table of Integrals, Series, and Products, 7th ed., edited by A. Jeffrey and D. Zwillinger (Academic Press, New York, 2007).

${ }^{31}$ J.-P. Gazeau, "From classical to quantum models: The regularising rôle of integrals, symmetry and probabilities," Found. Phys. 48, 1648-1667 (2018).

${ }^{32} \mathrm{H}$. Bergeron and J.-P. Gazeau, "Variations à la Fourier-Weyl-Wigner on quantizations of the plane and the half-plane," Entropy 20, 787-1-787-16 (2018).

${ }^{33}$ L. Cohen, "Generalized phase-space distribution functions," J. Math. Phys. 7, 781-786 (1966).

${ }^{34}$ L. Cohen, The Weyl Operator and its Generalization, Pseudo-Differential Operators: Theory and Applications Vol. 9 (Birkhaüser; Springer Basel, Heidelberg, 2013).

${ }^{35}$ B. S. Agarwal and E. Wolf, "Calculus for functions of noncommuting operators and general phase-space methods in quantum mechanics. I," Phys. Rev. D 2, 2161 (1970); "Calculus for functions of noncommuting operators and general phase-space methods in quantum mechanics. II," 2, 2187 (1970); "Calculus for functions of noncommuting operators and general phase-space methods in quantum mechanics. III," 2, 2206 (1970).

${ }^{36}$ S. T. Ali, N. M. Atakishiyev, S. M. Chumakov, and K. B. Wolf, “The Wigner function for general Lie groups and the Wavelet transform,” Ann. H. Poincaré 1, 685-714 (2000). 